\title{
New Herz Type Besov and Triebel-Lizorkin Spaces with Variable Exponents
}

\author{
Baohua Dong and Jingshi Xu \\ Department of Mathematics, Hainan Normal University, Haikou 571158, China \\ Correspondence should be addressed to Jingshi Xu, jingshixu@126.com
}

Received 19 October 2012; Accepted 3 December 2012

Academic Editor: Baoxiang Wang

Copyright (C) 2012 B. Dong and J. Xu. This is an open access article distributed under the Creative Commons Attribution License, which permits unrestricted use, distribution, and reproduction in any medium, provided the original work is properly cited.

The authors establish the boundedness of vector-valued Hardy-Littlewood maximal operator in Herz spaces with variable exponents. Then new Herz type Besov and Triebel-Lizorkin spaces with variable exponents are introduced. Finally, characterizations of these new spaces by maximal functions are given.

\section{Introduction}

Recent decades, variable exponent function spaces have attracted many attention. In fact, since the variable Lebesgue and Sobolev spaces were systemically studied by Kovác̆ik and Rákosník in [1], there are many spaces introduced, such as Bessel potential spaces with variable exponent, Besov and Trieble-Lizorkin spaces with variable exponents, and Morrey spaces with variable exponents; see [2-10] and reference therein. When the Hardy-Littlewood maximal operator is bounded on the variable Lebesgue spaces, many results in classic harmonic analysis and function theory are also hold for the variable exponent case; see [1113]. All mentioned spaces have many applications in differential equations, here we refer the readers to the survey [14].

Meanwhile, there are another type function spaces, Herz type spaces, have attracted many authors' interests for the last three decades. We recommend the readers to the monograph [15] and references therein. Indeed, many results in classic Lebesgue spaces have been generalized to Herz type spaces; see [16-20]. Herz type Besov and Triebel-Lizorkin spaces have been developed; see [21-24]. In 2010, Izuki in [25] introduced Herz space with variable exponent $\dot{K}_{p(\cdot)}^{\alpha, q}\left(\mathbb{R}^{n}\right)$ and $K_{p(\cdot)}^{\alpha, q}\left(\mathbb{R}^{n}\right)$ and proved the boundedness of sublinear operators on them. Then, in [26] Izuki obtained the boundedness of commutators on 
Herz spaces with variable exponent. In [27] Izuki established the boundedness of vectorvalued sublinear operators on Herz spaces with variable exponent $\dot{K}_{p(\cdot)}^{\alpha, q}\left(\mathbb{R}^{n}\right)$ and $K_{p(\cdot)}^{\alpha, q}\left(\mathbb{R}^{n}\right)$ and obtained equivalent norms and wavelet characterization of Herz-Sobolev spaces with variable exponent. In [28], Shi and the second author of this paper introduced Herz type Besov and Triebel-Lizorkin spaces with variable exponent, $\dot{K}_{p(\cdot)}^{\alpha, q}\left(\mathbb{R}^{n}\right) B_{\beta}^{s}$ and $K_{p(\cdot)}^{\alpha, q}\left(\mathbb{R}^{n}\right) B_{\beta}^{s}$ and $\dot{K}_{p(\cdot)}^{\alpha, q}\left(\mathbb{R}^{n}\right) F_{\beta}^{s}$ and $K_{p(\cdot)}^{\alpha, q}\left(\mathbb{R}^{n}\right) F_{\beta^{\prime}}^{s}$ and obtained their equivalent quasi-norms. Then, in [29], they gave a direct characterization of those spaces and its application. In 2012, Almeida and Drihem considered Herz spaces $\dot{K}_{p(\cdot)}^{\alpha(\cdot), q}\left(\mathbb{R}^{n}\right)$ and $K_{p(\cdot)}^{\alpha(\cdot), q}\left(\mathbb{R}^{n}\right)$ in [30], where the exponent $\alpha$ is variable as well, and gave boundedness results for a wide class of classical operators acting on such spaces.

Motivated by the previous papers, the goal of this paper is to introduce new Herz type Besov and Triebel-Lizorkin spaces with variable exponents. The structure of the paper is as follows. In Section 2 we will establish the boundedness of vector-valued Hardy-Littlewood maximal operator in spaces $\dot{K}_{p(\cdot)}^{\alpha(\cdot), q}\left(\mathbb{R}^{n}\right)$ and $K_{p(\cdot)}^{\alpha(\cdot), q}\left(\mathbb{R}^{n}\right)$. In Section 3 we give characterizations of these Herz type Besov and Triebel-Lizorkin spaces with variable exponents by maximal functions.

\section{Vector-Valued Estimates}

In this section we will establish vector-valued estimates of Hardy-Littlewood maximal operator in Herz spaces with variable exponents. Before stating our result, we recall some definitions and notations. First, we give some convention. Throughout this paper $|S|$ denotes the Lebesgue measure and $\chi_{S}$ the characteristics function for a measurable set $S \subset \mathbb{R}^{n}$. We also use the notation $a \lesssim b$ if there exists a constant $c>0$ such that $a \leqslant c b$. If $a \lesssim b$ and $b \lesssim a$ we will write $a \approx b$. Finally we claim that $C$ is always a positive constant but it may change from line to line.

Definition 2.1. Let $E$ be a measurable set in $\mathbb{R}^{n}$ with $|E|>0$. Let $p(\cdot): E \rightarrow[1, \infty)$ be a measurable function. Denote

$$
L^{p(\cdot)}(E):=\left\{f \text { is measurable on } E: \rho_{p(\cdot)}\left(\frac{f}{\lambda}\right)<\infty \text { for some constant } \lambda>0\right\}
$$

where $\rho_{p(\cdot)}(f):=\int_{E}|f(x)|^{p(x)} d x$, and

$$
\|f\|_{L^{p(\cdot)}(E)}:=\inf \left\{\lambda>0: \rho_{p(\cdot)}\left(\frac{f}{\lambda}\right) \leqslant 1\right\}
$$

Then $L^{p(\cdot)}(E)$ is a Banach space with the norm $\|\cdot\|_{L^{p(\cdot)}(E)}$.

Let $L_{\text {loc }}^{1}\left(\mathbb{R}^{n}\right)$ be the collection of all locally integrable functions on $\mathbb{R}^{n}$. Given a function $f \in L_{\text {loc }}^{1}\left(\mathbb{R}^{n}\right)$, the Hardy-Littlewood maximal operator $\mathcal{M}$ is defined by

$$
\mathcal{M} f(x):=\sup _{r>0} r^{-n} \int_{B(x, r)}|f(y)| d y, \quad \forall x \in \mathbb{R}^{n}
$$


where $B(x, r):=\left\{y \in \mathbb{R}^{n}:|x-y|<r\right\}$. We also use the following notation: $p_{-}:=\operatorname{ess} \inf \{p(x)$ : $\left.x \in \mathbb{R}^{n}\right\}$ and $p_{+}:=\operatorname{ess} \sup \left\{p(x): x \in \mathbb{R}^{n}\right\}$. The set $p\left(\mathbb{R}^{n}\right)$ consists of all $p(\cdot)$ satisfying $p_{-}>1$ and $p_{+}<\infty . B\left(\mathbb{R}^{n}\right)$ is the set of $p(\cdot) \in D\left(\mathbb{R}^{n}\right)$ satisfying the condition that $\mathcal{M}$ is bounded on $L^{p(\cdot)}\left(\mathbb{R}^{n}\right)$. It is well known that if $p(\cdot) \in \mathcal{D}\left(\mathbb{R}^{n}\right)$ then $p(\cdot) \in \mathbb{B}\left(\mathbb{R}^{n}\right)$ is equivalent to $p^{\prime}(\cdot) \in \mathbb{B}\left(\mathbb{R}^{n}\right)$, where $p^{\prime}(\cdot)$ is the conjugate exponent to $p(\cdot)$, that means $p^{\prime}(\cdot)=p(\cdot) /(p(\cdot)-1)$; see [12]. For more about the set $B\left(\mathbb{R}^{n}\right)$, one can see $[11,12,31,32]$. Moreover, we define $p^{0}\left(\mathbb{R}^{n}\right)$ to be the set of measurable functions $p$ on $\mathbb{R}^{n}$ with the range in $(0, \infty)$ such that $p_{-}:=\operatorname{ess} \inf \{p(x)$ : $\left.x \in \mathbb{R}^{n}\right\}>0$ and $p_{+}:=$ess $\sup \left\{p(x): x \in \mathbb{R}^{n}\right\}<\infty$. Given $p(\cdot) \in p^{0}\left(\mathbb{R}^{n}\right)$, one can define the space $L^{p(\cdot)}\left(\mathbb{R}^{n}\right)$ as in Definition 2.1. This is equivalent to defining it to be the set of all functions $f$ such that $|f|^{p_{0}} \in L^{p(\cdot) / p_{0}}\left(\mathbb{R}^{n}\right)$, where $0<p_{0}<p_{-}$and $p(\cdot) / p_{0} \in D\left(\mathbb{R}^{n}\right)$. We also define a quasi-norm on this space by $\|f\|_{L^{p())}\left(\mathbb{R}^{n}\right)}=\left\||f|^{p_{0}}\right\|_{L^{p(\cdot) / p_{0}\left(\mathbb{R}^{n}\right)}}^{1 / p_{0}}$.

Lemma 2.2 (see [25]). Let $p(\cdot) \in \mathbb{B}\left(\mathbb{R}^{n}\right)$. Then there exist $0<\delta_{1}, \delta_{2}<1$ depending only on $p(\cdot)$ and $n$ such that for balls $B$ in $\mathbb{R}^{n}$ and all measurable subsets $S \subset B$,

$$
\frac{\left\|X_{S}\right\|_{L^{p(\cdot)\left(\mathbb{R}^{n}\right)}}}{\left\|X_{B}\right\|_{L^{p(\cdot)}\left(\mathbb{R}^{n}\right)}} \leqslant C\left(\frac{|S|}{|B|}\right)^{\delta_{1}}, \quad \frac{\left\|X_{S}\right\|_{L^{p^{\prime}(\cdot)\left(\mathbb{R}^{n}\right)}}}{\left\|\chi_{B}\right\|_{L^{p^{\prime}(\cdot)}\left(\mathbb{R}^{n}\right)}} \leqslant C\left(\frac{|S|}{|B|}\right)^{\delta_{2}} .
$$

Lemma 2.3 (see [25]). Let $p(\cdot) \in \mathbb{B}\left(\mathbb{R}^{n}\right)$. Then there exists a positive constant $C>0$ such that for balls $B$ in $\mathbb{R}^{n}$,

$$
\frac{1}{|B|}\left\|X_{B}\right\|_{L^{p(\cdot)}\left(\mathbb{R}^{n}\right)}\left\|X_{B}\right\|_{L^{p^{(\cdot)}\left(\mathbb{R}^{n}\right)}} \leqslant C
$$

Lemma 2.4 (see [11]). Let $p(\cdot) \in \mathbb{B}\left(\mathbb{R}^{n}\right)$ and $1<r<\infty$, then there exists a positive constant $C$ such that for all sequences $\left\{f_{j}\right\}_{j=1}^{\infty}$ of locally integrable functions on $\mathbb{R}^{n}$,

$$
\left\|\left(\sum_{j=1}^{\infty}\left|\mathcal{M} f_{j}\right|^{r}\right)^{1 / r}\right\|_{L^{p(\cdot)}} \leqslant C\left\|\left(\sum_{j=1}^{\infty}\left|f_{j}\right|^{r}\right)^{1 / r}\right\|_{L^{p(\cdot)}} .
$$

For giving the definition of the Herz spaces with variable exponent, let us introduce the following notations. Let $k \in \mathbb{Z} . B_{k}:=\left\{x \in \mathbb{R}^{n}:|x| \leqslant 2^{k}\right\} . R_{k}:=B_{k} \backslash B_{k-1} \cdot \chi_{k}:=\chi_{R_{k}}$.

$\mathbb{N}_{0}$ denotes the set of all nonnegative integers. For $m \in \mathbb{N}_{0}$, we denote $\tilde{X}_{m}:=\chi_{R_{m}}$ if $m \geq 1$ and $\tilde{X}_{0}:=\chi_{B_{0}}$.

Definition 2.5. Let $0<q \leqslant \infty, p(\cdot) \in D\left(\mathbb{R}^{n}\right)$ and $\alpha(\cdot): \mathbb{R}^{n} \rightarrow \mathbb{R}$ with $\alpha(\cdot) \in L^{\infty}\left(\mathbb{R}^{n}\right)$.

(i) The homogeneous Herz space $\dot{K}_{p(\cdot)}^{\alpha(\cdot), q}\left(\mathbb{R}^{n}\right)$ is defined by

$$
\dot{K}_{p(\cdot)}^{\alpha(\cdot), q}\left(\mathbb{R}^{n}\right):=\left\{f \in L_{\mathrm{loc}}^{p(\cdot)}\left(\mathbb{R}^{n} \backslash 0\right):\|f\|_{\dot{K}_{p(\cdot)}^{\alpha(\cdot), q}\left(\mathbb{R}^{n)}\right.}<\infty\right\}
$$


where

$$
\|f\|_{K_{p(\cdot)}^{\alpha(\cdot), q}\left(\mathbb{R}^{n}\right)}:=\left\{\sum_{k=-\infty}^{\infty}\left\|2^{\alpha(\cdot) k} f X_{k}\right\|_{L^{p(\cdot)}\left(\mathbb{R}^{n}\right)}^{q}\right\}^{1 / q} .
$$

(ii) The nonhomogeneous Herz space $K_{p(\cdot)}^{\alpha(\cdot), q}\left(\mathbb{R}^{n}\right)$ is defined by

$$
K_{p(\cdot)}^{\alpha(\cdot), q}\left(\mathbb{R}^{n}\right):=\left\{f \in L_{\mathrm{loc}}^{p(\cdot)}\left(\mathbb{R}^{n}\right):\|f\|_{K_{p(\cdot)}^{\alpha(\cdot), q}\left(\mathbb{R}^{n}\right)}<\infty\right\}
$$

where

$$
\|f\|_{K_{p(\cdot)}^{\alpha(\cdot),\left(\mathbb{R}^{n}\right)}}:=\left\{\sum_{m=0}^{\infty}\left\|2^{\alpha(\cdot) m} f \tilde{X}_{m}\right\|_{L^{p(\cdot)}\left(\mathbb{R}^{n}\right)}^{q}\right\}^{1 / q} .
$$

Definition 2.6. A function $\alpha(\cdot): \mathbb{R}^{n} \rightarrow \mathbb{R}$ is called locally log-Hölder continuous on $\mathbb{R}^{n}$ if there exists $c_{\log }>0$ such that

$$
|\alpha(x)-\alpha(y)| \leqslant \frac{c_{\log }}{\log (e+1 /|x-y|)}
$$

for all $x, y \in \mathbb{R}^{n}$. If $|\alpha(x)-\alpha(0)| \leqslant c_{\log } / \log (e+1 /|x|)$ for all $x \in \mathbb{R}^{n}$, then $\alpha(\cdot)$ is called logHölder continuous at origin. $\alpha(\cdot)$ is called satisfying the log-Hölder decay condition if there exist $\alpha_{\infty} \in \mathbb{R}$ and a constant $c_{\log }>0$ such that

$$
\left|\alpha(x)-\alpha_{\infty}\right| \leqslant \frac{c_{\log }}{\log (e+|x|)}
$$

for all $x \in \mathbb{R}^{n}$.

By $\ell^{q}, q \in(0, \infty]$, we denote the discrete Lebesgue space equipped with the usual quasi-norm. Let us denote

$$
\left\|\left\{g_{k}\right\}\right\|_{\ell_{>}^{q}\left(L^{p(\cdot)}\right)}:=\left(\sum_{k=0}^{\infty}\left\|g_{k}\right\|_{p(\cdot)}^{q}\right)^{1 / q}, \quad\left\|\left\{g_{k}\right\}\right\|_{\ell_{<}^{q}\left(L^{p(\cdot)}\right)}:=\left(\sum_{k=-\infty}^{-1}\left\|g_{k}\right\|_{p(\cdot)}^{q}\right)^{1 / q},
$$

for sequences $\left\{g_{k}\right\}_{k \in \mathbb{Z}}$ of measurable functions (with the usual modification when $q=\infty$.)

Proposition 2.7. Let $\alpha(\cdot) \in L^{\infty}\left(\mathbb{R}^{n}\right), p(\cdot) \in p\left(\mathbb{R}^{n}\right)$ and $q \in(0, \infty]$. If $\alpha(\cdot)$ satisfies log-Hölder decay condition at infinity, then

$$
K_{p(\cdot)}^{\alpha(\cdot), q}\left(\mathbb{R}^{n}\right)=K_{p(\cdot)}^{\alpha_{\infty}, q}\left(\mathbb{R}^{n}\right)
$$


Journal of Function Spaces and Applications

Additionally, if $\alpha(\cdot)$ is log-Hölder continuous at the origin, then

$$
\|f\|_{\dot{K}_{p(\cdot)}^{\alpha(\cdot), q}\left(\mathbb{R}^{n)}\right.} \approx\left\|\left\{2^{k \alpha(0)} f X_{k}\right\}\right\|_{\ell_{<}^{q}\left(L^{p(\cdot)}\right)}+\left\|\left\{2^{k \alpha_{\infty}} f X_{k}\right\}\right\|_{\ell_{>}^{q}\left(L^{p(\cdot)}\right)}
$$

We have the boundedness of vector-valued Hardy-Littlewood maximal operator in Herz spaces with variable exponents, which generalizes the results in [27, 33].

Theorem 2.8. Let $p(\cdot) \in \mathbb{B}\left(\mathbb{R}^{n}\right), 0<q<\infty, \alpha(\cdot): \mathbb{R}^{n} \rightarrow \mathbb{R}$ with $\alpha(\cdot) \in L^{\infty}\left(\mathbb{R}^{n}\right)$ and $\alpha(\cdot)$ be $\log$-Hölder continuous, both at the origin and at infinity, such that $-n \delta_{1}<\alpha(0) \leqslant \alpha_{\infty}<n \delta_{2}$, where $0<\delta_{1}, \delta_{2}<1$ are constants satisfying (2.4), $1<r<\infty$, then there is a constant $C$ independent of sequences $\left\{f_{j}\right\}_{j=1}^{\infty}$ of locally integrable functions on $\mathbb{R}^{n}$ such that

$$
\begin{aligned}
& \left\|\left(\sum_{k=1}^{\infty}\left|\mathcal{M} f_{k}\right|^{r}\right)^{1 / r}\right\|_{\dot{K}_{p(\cdot)}^{\alpha(\cdot), q\left(\mathbb{R}^{n}\right)}} \leqslant C\left\|\left(\sum_{k=1}^{\infty}\left|f_{k}\right|^{r}\right)^{1 / r}\right\|_{\dot{K}_{p(\cdot)}^{\alpha(\cdot), q\left(\mathbb{R}^{n}\right)}}, \\
& \left\|\left(\sum_{k=1}^{\infty}\left|\mathcal{M} f_{k}\right|^{r}\right)^{1 / r}\right\|_{K_{p(\cdot)}^{\alpha(\cdot), q\left(\mathbb{R}^{n}\right)}} \leqslant C\left\|\left(\sum_{k=1}^{\infty}\left|f_{k}\right|^{r}\right)^{1 / r}\right\|_{K_{p(\cdot)}^{\alpha(\cdot), q\left(\mathbb{R}^{n}\right)}} .
\end{aligned}
$$

Proof. Here we only prove the result for homogeneous Herz space, for nonhomogeneous Herz space it can be proved by similar way. We only consider sequences $\left\{f_{j}\right\}_{j=1}^{\infty}$ of locally measurable functions on $\mathbb{R}^{n}$ such that $\left(\sum_{k=1}^{\infty}\left|f_{k}\right|^{r}\right)^{1 / r} \in \dot{K}_{p(\cdot)}^{\alpha(\cdot), q}\left(\mathbb{R}^{n}\right)$.

Firstly, by Proposition 2.7 we have

$$
\begin{aligned}
&\left\|\left(\sum_{j=1}^{\infty}\left|\mathcal{M} f_{j}\right|^{r}\right)^{1 / r}\right\|_{\dot{K}_{p(\cdot)}^{\alpha(\cdot), q}\left(\mathbb{R}^{n}\right)} \approx\left\|\left\{2^{k \alpha(0)}\left(\sum_{j=1}^{\infty}\left|\mathcal{M} f_{j}\right|^{r}\right)^{1 / r} x_{k}\right\}\right\|_{\ell_{<}^{q}\left(L^{p(\cdot)}\right)} \\
&+\left\|\left\{2^{k \alpha_{\infty}}\left(\sum_{j=1}^{\infty}\left|\mathcal{M} f_{j}\right|^{r}\right)^{1 / r} x_{k}\right\}\right\|_{\ell_{>}^{q}\left(L^{p(\cdot)}\right)} \\
&:=A_{<}+A_{>} .
\end{aligned}
$$


To continue, we let $f_{j}^{i}=\chi_{i} f_{j}$. Now we estimate $A_{<}$by Minkowski inequality as follows:

$$
\begin{aligned}
& A_{<}:=\left\{\sum_{k=-\infty}^{-1} 2^{\alpha(0) q k}\left\|X_{k}\left(\sum_{j=1}^{\infty}\left|\mathcal{M} f_{j}\right|^{r}\right)^{1 / r}\right\|_{L^{p(\cdot)}}^{q}\right\}^{1 / q} \\
& =\left\{\sum_{k=-\infty}^{-1} 2^{\alpha(0) q k}\left\|X_{k}\left(\sum_{j=1}^{\infty}\left|\mathcal{M} \sum_{i=-\infty}^{\infty} f_{j}^{i}\right|^{r}\right)^{1 / r}\right\|_{L^{p(\cdot)}}^{q}\right\}^{1 / q} \\
& \leqslant\left\{\sum_{k=-\infty}^{-1} 2^{\alpha(0) q k}\left\|X_{k} \sum_{i=-\infty}^{\infty}\left(\sum_{j=1}^{\infty}\left|\mathcal{M} f_{j}^{i}\right|^{r}\right)^{1 / r}\right\|_{L^{p(\cdot)}}^{q}\right\}^{1 / q} \\
& \lesssim\left\{\sum_{k=-\infty}^{-1} 2^{\alpha(0) q k}\left\|X X k_{i=-\infty}^{k-2}\left(\sum_{j=1}^{\infty}\left|\mathcal{M} f_{j}^{i}\right|^{r}\right)^{1 / r}\right\|_{L^{p(\cdot)}}^{q}\right\}^{1 / q} \\
& +\left\{\sum_{k=-\infty}^{-1} 2^{\alpha(0) q k}\left\|x k \sum_{i=k-1}^{k+1}\left(\sum_{j=1}^{\infty}\left|\mathcal{M} f_{j}^{i}\right|^{r}\right)^{1 / r}\right\|_{L^{p(\cdot)}}^{q}\right\}^{1 / q} \\
& +\left\{\sum_{k=-\infty}^{-1} 2^{\alpha(0) q k}\left\|X k \sum_{i=k+2}^{\infty}\left(\sum_{j=1}^{\infty}\left|\mathcal{M} f_{j}^{i}\right|^{r}\right)^{1 / r}\right\|_{L^{p(\cdot)}}^{q}\right\}^{1 / q} \\
& :=I_{<}+I I_{<}+I I I_{<} \text {. }
\end{aligned}
$$

By the same way we consider $A_{>}$,

$$
\begin{aligned}
A_{>} \lesssim & \left\{\sum_{k=0}^{\infty} 2^{\alpha_{\infty} q k}\left\|X_{k} \sum_{i=-\infty}^{k-2}\left(\sum_{j=1}^{\infty}\left|\mathcal{M} f_{j}^{i}\right|^{r}\right)^{1 / r}\right\|_{L^{p(\cdot)}}^{q}\right\}^{1 / q} \\
& +\left\{\sum_{k=0}^{\infty} 2^{\alpha_{\infty} q k}\left\|X X k_{k} \sum_{i=k-1}^{k+1}\left(\sum_{j=1}^{\infty}\left|\mathcal{M} f_{j}^{i}\right|^{r}\right)^{1 / r}\right\|_{L^{p(\cdot)}}^{q}\right\}^{1 / q} \\
& +\left\{\sum_{k=0}^{\infty} 2^{\alpha_{\infty} q k}\left\|X_{k} \sum_{i=k+2}^{\infty}\left(\sum_{j=1}^{\infty}\left|\mathcal{M} f_{j}^{i}\right|^{r}\right)^{1 / r}\right\|_{L^{p(\cdot)}}^{q}\right\}^{1 / q} \\
:= & I_{>}+I I_{>}+I I I_{>} .
\end{aligned}
$$


Journal of Function Spaces and Applications

So we get

$$
\left\|\left(\sum_{j=1}^{\infty}\left|\mathcal{M} f_{j}\right|^{r}\right)^{1 / r}\right\|_{\dot{K}_{p(\cdot)}^{\alpha(\cdot), q\left(\mathbb{R}^{n}\right)}} \lesssim I+I I+I I I,
$$

with $I:=I_{<}+I_{>}, I I:=I I_{<}+I I_{>}, I I I:=I I I_{<}+I I I_{>}$.

Secondly, we divide the proof into three steps.

Step 1. We consider II. By Lemma 2.4 we have

$$
\begin{aligned}
& I I_{<} \lesssim\left\{\sum_{k=-\infty}^{-1} 2^{\alpha(0) q k} \sum_{i=k-1}^{k+1}\left\|\left(\sum_{j=1}^{\infty}\left|f_{j}^{i}\right|^{r}\right)^{1 / r}\right\|_{L^{p(\cdot)}}^{q}\right\}^{1 / q} \\
& \lesssim\left\{\sum_{k=-\infty}^{-1} 2^{\alpha(0) q k} \sum_{i=k-1}^{k+1}\left\|X_{i}\left(\sum_{j=1}^{\infty}\left|f_{j}\right|^{r}\right)^{1 / r}\right\|_{L^{p(\cdot)}}^{q}\right\}^{1 / q} \\
& \lesssim\left\{\sum_{k=-\infty}^{-1} 2^{\alpha(0) q k}\left\|X_{k-1}\left(\sum_{j=1}^{\infty}\left|f_{j}\right|^{r}\right)^{1 / r}\right\|_{L^{p(\cdot)}}^{q}\right\}^{1 / q}+\left\{\sum_{k=-\infty}^{-1} 2^{\alpha(0) q k}\left\|X_{k}\left(\sum_{j=1}^{\infty}\left|f_{j}\right|^{r}\right)^{1 / r}\right\|_{L^{p(\cdot)}}^{q}\right\}^{1 / q} \\
& +\left\{\sum_{k=-\infty}^{-1} 2^{\alpha(0) q k} \| X_{k+1}\left(\sum_{j=1}^{\infty}\left|f_{j}\right|^{r} \|_{L^{p(\cdot)}}^{1 / r}\right\}^{q}\right. \\
& \lesssim\left\{\sum_{k=-\infty}^{-2} 2^{\alpha(0) q k}\left\|X_{k}\left(\sum_{j=1}^{\infty}\left|f_{j}\right|^{r}\right)^{1 / r}\right\|_{L^{p(\cdot)}}^{q}\right\}^{1 / q}+\left\|\left\{2^{\alpha(0) k}\left(\sum_{j=1}^{\infty}\left|f_{j}\right|^{r}\right)^{1 / r} X_{k}\right\}\right\|_{\ell_{<}^{q}\left(L^{p(\cdot)}\right)} \\
& +\left\{\sum_{k=-\infty}^{0} 2^{\alpha(0) q k}\left\|X_{k}\left(\sum_{j=1}^{\infty}\left|f_{j}\right|^{r}\right)^{1 / r}\right\|_{L^{p(\cdot)}}^{q}\right\}^{1 / q} \\
& \lesssim\left\|\left\{2^{\alpha(0) k}\left(\sum_{j=1}^{\infty}\left|f_{j}\right|^{r}\right)^{1 / r} x_{k}\right\}\right\|_{\ell_{<}^{q}\left(L^{p(\cdot)}\right)} .
\end{aligned}
$$


Similarly, we have

$$
\begin{aligned}
I I_{>} \lesssim & \left\{\sum_{k=-1}^{\infty} 2^{\alpha_{\infty} q k}\left\|X X_{k}\left(\sum_{j=1}^{\infty}\left|f_{j}\right|^{r}\right)^{1 / r}\right\|_{L^{p(\cdot)}}^{q}\right\}^{1 / q}+\left\|\left\{2^{\alpha_{\infty} k}\left(\sum_{j=1}^{\infty}\left|f_{j}\right|^{r}\right)^{1 / r} X_{k}\right\}\right\|_{\ell_{(}^{q}\left(L^{p(\cdot)}\right)} \\
& +\left\{\sum_{k=1}^{\infty} 2^{\alpha_{\infty} q k}\left\|X_{k}\left(\sum_{j=1}^{\infty}\left|f_{j}\right|^{r}\right)^{1 / r}\right\|_{L^{p(\cdot)}}^{q}\right\}^{1 / q} \\
\lesssim & \left\|\left\{2^{\alpha_{\infty} k}\left(\sum_{j=1}^{\infty}\left|f_{j}\right|^{r}\right)^{1 / r} x_{k}\right\}\right\|_{\mathcal{\ell}_{(}^{q}\left(L^{p(\cdot)}\right)} .
\end{aligned}
$$

Thus

$$
I I:=I I_{<}+I I_{>} \lesssim\left\|\left(\sum_{j=1}^{\infty}\left|f_{j}\right|^{r}\right)^{1 / r}\right\|_{\dot{K}_{p(\cdot)}^{\alpha(\cdot), q}}
$$

Step 2. We consider I. We recall that the Hardy-Littlewood maximal operator satisfies the size condition

$$
M f(x) \leqslant C \int_{\mathbb{R}^{n}}|x-y|^{-n}|f(y)| d y
$$

for all $f \in L_{\text {loc }}^{1}\left(\mathbb{R}^{n}\right)$ with compact support and a.e $x \notin \operatorname{supp} f$.

Therefore, for for all $i \leqslant k-2, x \in R_{k}, 1<r<\infty$, by the size condition and the generalized Minkowski inequality, we obtain

$$
\begin{aligned}
\left(\sum_{j=1}^{\infty} \mathcal{M}^{r}\left(f_{j}^{i}\right)(x)\right)^{1 / r} & \lesssim\left[\sum_{j=1}^{\infty}\left(2^{-k n} \int_{\mathbb{R}^{n}}\left|f_{j}^{i}(y)\right| d y\right)^{r}\right]^{1 / r} \\
& \lesssim 2^{-k n} \int_{\mathbb{R}^{n}}\left(\sum_{j=1}^{\infty}\left|f_{j}^{i}\right|^{r}\right)^{1 / r} d y
\end{aligned}
$$


Journal of Function Spaces and Applications

By Hölder's inequality we get

$$
\begin{aligned}
I_{<} & \lesssim\left\{\sum_{k=-\infty}^{-1} 2^{\alpha(0) q k}\left\|X_{k} \sum_{i=-\infty}^{k-2} 2^{-k n} \int_{\mathbb{R}^{n}}\left(\sum_{j=1}^{\infty}\left|f_{j}^{i}\right|^{r}\right)^{1 / r} d y\right\|_{L^{p(\cdot)}}^{q}\right\}^{1 / q} \\
& \approx\left\{\sum_{k=-\infty}^{-1} 2^{\alpha(0) q k}\left\|X_{k}\right\|_{L^{p(\cdot)}}^{q}\left(\sum_{i=-\infty}^{k-2} 2^{-k n} \int_{\mathbb{R}^{n}}\left(\sum_{j=1}^{\infty}\left|f_{j}^{i}\right|^{r}\right)^{1 / r} d y\right)^{q}\right\}^{1 / q} \\
& \lesssim\left\{\sum_{k=-\infty}^{-1} 2^{\alpha(0) q k}\left\|X_{k}\right\|_{L^{p(\cdot)}}^{q} \times\left(\sum_{i=-\infty}^{k-2} 2^{-k n}\left\|\left(\sum_{j=1}^{\infty}\left|f_{j}\right|^{r}\right)^{1 / r} x_{i}\right\|_{L^{p(\cdot)}}\left\|X_{i}\right\|_{L^{p^{(\cdot)}}}\right)^{q}\right\}^{1 / q} .
\end{aligned}
$$

On the other hand, by using Lemmas 2.2 and 2.3, we have

$$
2^{-k n}\left\|X_{X}\right\|_{L^{p^{(\cdot)}}}\left\|X_{X_{i}}\right\|_{L^{p^{\prime}(\cdot)}} \lesssim 2^{-k n}\left\|X_{B_{i}}\right\|_{L^{p^{\prime}(\cdot)}}\left|B_{k}\right|\left\|_{X_{B_{k}}}\right\|_{L^{p^{\prime}(\cdot)}}^{-1} \lesssim 2^{n \delta_{2}(i-k)} .
$$

We put (2.27) into (2.26) and get

$$
\begin{aligned}
I_{<} & \lesssim\left\{\sum_{k=-\infty}^{-1} 2^{\alpha(0) k q}\left(\sum_{i=-\infty}^{k-2}\left\|\left(\sum_{j=1}^{\infty}\left|f_{j}\right|^{r}\right)^{1 / r} x_{i}\right\|_{L^{p(\cdot)}} 2^{n \delta_{2}(i-k)}\right)^{q}\right\}^{1 / q} \\
& \approx\left\{\sum_{k=-\infty}^{-1}\left(\sum_{i=-\infty}^{k-2} 2^{\alpha(0) k}\left\|\left(\sum_{j=1}^{\infty}\left|f_{j}\right|^{r}\right)^{1 / r} x_{i}\right\|_{L^{p(\cdot)}} 2^{n \delta_{2}(i-k)}\right)^{q}\right\}^{1 / q} \\
& \approx\left\{\sum_{k=-\infty}^{-1}\left(\sum_{i=-\infty}^{k-2} 2^{\alpha(0) i}\left\|\left(\sum_{j=1}^{\infty}\left|f_{j}\right|^{r}\right)^{1 / r} x_{i}\right\|_{L^{p(\cdot)}} 2^{b(i-k)}\right)^{q}\right\}^{1 / q},
\end{aligned}
$$

here $b=n \delta_{2}-\alpha(0)>0$. 
For $1<q<\infty$, we can make (2.28) further calculation by Hölder's inequality

$$
\begin{aligned}
I_{<} & \lesssim\left\{\sum_{k=-\infty}^{-1}\left(\sum_{i=-\infty}^{k-2} 2^{\alpha(0) i q}\left\|\left(\sum_{j=1}^{\infty}\left|f_{j}\right|^{r}\right)^{1 / r} x_{i}\right\|_{L^{p \cdot()}}^{q} 2^{b q(i-k) / 2}\right)\left(\sum_{i=-\infty}^{k-2} 2^{b q^{\prime}(i-k) / 2}\right)^{q / q^{\prime}}\right\}^{1 / q} \\
& \lesssim\left\{\sum_{k=-\infty}^{-1} \sum_{i=-\infty}^{k-2} 2^{\alpha(0) i q}\left\|\left(\sum_{j=1}^{\infty}\left|f_{j}\right|^{r}\right)^{1 / r} x_{i}\right\|_{L^{p(\cdot)}}^{q} 2^{b q(i-k) / 2}\right\}^{1 / q} \\
& \approx\left\{\sum_{i=-\infty}^{-3} 2^{\alpha(0) i q}\left\|\left(\sum_{j=1}^{\infty}\left|f_{j}\right|^{r}\right)^{1 / r} x_{i}\right\|_{L^{p(\cdot)}}^{q} \sum_{k=i+2}^{-1} 2^{b q(i-k) / 2}\right\}^{1 / q} \\
& \lesssim\left\{\sum_{i=-\infty}^{-3} 2^{\alpha(0) i q}\left\|\left(\sum_{j=1}^{\infty}\left|f_{j}\right|^{r}\right)^{1 / r} x_{i}\right\|_{L^{p()}}^{q}\right\}^{1 / q} \\
& \lesssim\left\|\left\{2^{\alpha(0) k}\left(\sum_{j=1}^{\infty}\left|f_{j}\right|^{r}\right)^{1 / r} x_{k}\right\}\right\|_{\ell_{(}^{q}\left(L^{p(\cdot)}\right)}
\end{aligned}
$$

For $0<q \leqslant 1$, we have

$$
\begin{aligned}
I_{<} & \lesssim\left\{\sum_{k=-\infty}^{-1} \sum_{i=-\infty}^{k-2} 2^{\alpha(0) i q} \|\left(\sum_{j=1}^{\infty}\left|f_{j}\right|^{r} x^{1 / r} x_{i} \|_{L^{p(\cdot)}}^{q} 2^{b q(i-k)}\right\}^{1 / q}\right. \\
& \approx\left\{\sum_{i=-\infty}^{-3} 2^{\alpha(0) i q}\left\|\left(\sum_{j=1}^{\infty}\left|f_{j}\right|^{r}\right)^{1 / r} x_{i}\right\|_{L^{p(\cdot)}}^{q} \sum_{k=i+2}^{-1} 2^{b q(i-k)}\right\}^{1 / q} \\
& \lesssim\left\{\sum_{i=-\infty}^{-3} 2^{\alpha(0) i q}\left\|\left(\sum_{j=1}^{\infty}\left|f_{j}\right|^{r}\right)^{1 / r} x_{i}\right\|_{L^{p(\cdot)}}^{q}\right\}^{1 / q} \\
& \lesssim\left\|\left\{2^{\alpha(0) k}\left(\sum_{j=1}^{\infty}\left|f_{j}\right|^{r}\right)^{1 / r} x_{k}\right\}\right\|_{\mathcal{R}_{(}^{q}\left(L^{p(\cdot)}\right)} .
\end{aligned}
$$


Journal of Function Spaces and Applications

Similarly, we have

$$
I_{>} \lesssim\left\{\sum_{k=0}^{\infty}\left(\sum_{i=-\infty}^{k-2} 2^{\alpha_{\infty} i}\left\|\left(\sum_{j=1}^{\infty}\left|f_{j}\right|^{r}\right)^{1 / r} x_{i}\right\|_{L^{p(\cdot)}} 2^{b(i-k)}\right)^{q}\right\}^{1 / q},
$$

here $b=n \delta_{2}-\alpha_{\infty}>0$.

For $1<q<\infty$, we have

$$
\begin{aligned}
& I_{>} \lesssim\left\{\sum_{k=0}^{\infty} \sum_{i=-\infty}^{k-2} 2^{\alpha_{\infty} i q}\left\|\left(\sum_{j=1}^{\infty}\left|f_{j}\right|^{r}\right)^{1 / r} x_{i}\right\|_{L^{p(\cdot)}}^{q} 2^{b q(i-k) / 2}\right\}^{1 / q} \\
& \approx\left\{\sum_{i=-\infty}^{-2} 2^{\alpha_{\infty} i q}\left\|\left(\sum_{j=1}^{\infty}\left|f_{j}\right|^{r}\right)^{1 / r} x_{i}\right\|_{L^{p(\cdot)}}^{q} \sum_{k=0}^{\infty} 2^{b q(i-k) / 2}\right. \\
& \left.+\sum_{i=-1}^{\infty} 2^{\alpha_{\infty} i q}\left\|\left(\sum_{j=1}^{\infty}\left|f_{j}\right|^{r}\right)^{1 / r} x_{i}\right\|_{L^{p(\cdot)}}^{q} \sum_{k=i+2}^{\infty} 2^{b q(i-k) / 2}\right\}^{1 / q} \\
& \lesssim\left\{\sum_{i=-\infty}^{-2} 2^{\left(\alpha_{\infty}+n \delta_{2}\right) i q / 2}\left\|\left(\sum_{j=1}^{\infty}\left|f_{j}\right|^{r}\right)^{1 / r} x_{i}\right\|_{L^{p(\cdot)}}^{q}+\sum_{i=-1}^{\infty} 2^{\alpha_{\infty} i q}\left\|\left(\sum_{j=1}^{\infty}\left|f_{j}\right|^{r}\right)^{1 / r} x_{i}\right\|_{L^{p(\cdot)}}^{q}\right\}^{1 / q} \\
& \lesssim\left\{\sum_{i=-\infty}^{-2} 2^{\alpha(0) i q+\left(\left(\alpha_{\infty}-2 \alpha(0)+n \delta_{2}\right) i q\right) / 2}\left\|\left(\sum_{j=1}^{\infty}\left|f_{j}\right|^{r}\right)^{1 / r} x_{i}\right\|_{L^{p(\cdot)}}^{q}\right. \\
& \left.+\sum_{i=-1}^{\infty} 2^{\alpha_{\infty} i q}\left\|\left(\sum_{j=1}^{\infty}\left|f_{j}\right|^{r}\right)^{1 / r} x_{i}\right\|_{L^{p(\cdot)}}^{q}\right\}^{1 / q} \\
& \lesssim\left\{\sum_{i=-\infty}^{-2} 2^{\alpha(0) i q}\left\|\left(\sum_{j=1}^{\infty}\left|f_{j}\right|^{r}\right)^{1 / r} x_{i}\right\|_{L^{p(\cdot)}}^{q}+\sum_{i=-1}^{\infty} 2^{\alpha_{\infty} i q}\left\|\left(\sum_{j=1}^{\infty}\left|f_{j}\right|^{r}\right)^{1 / r} x_{i}\right\|_{L^{p(\cdot)}}^{q}\right\}^{1 / q} \\
& \lesssim\left\|\left\{2^{\alpha(0) k}\left(\sum_{j=1}^{\infty}\left|f_{j}\right|^{r}\right)^{1 / r} X_{k}\right\}\right\|_{\ell_{<}^{q}\left(L^{p())}\right)}+\left\|\left\{2^{\alpha_{\infty} k}\left(\sum_{j=1}^{\infty}\left|f_{j}\right|^{r}\right)^{1 / r} X_{k}\right\}\right\|_{\ell_{>}^{q}\left(L^{p()}\right)} .
\end{aligned}
$$


For $0<q \leqslant 1$, we have

$$
\begin{aligned}
& I_{>} \lesssim\left\{\sum_{k=0}^{\infty} \sum_{i=-\infty}^{k-2} 2^{\alpha_{\infty} i q}\left\|\left(\sum_{j=1}^{\infty}\left|f_{j}\right|^{r}\right)^{1 / r} X_{i}\right\|_{L^{p(\cdot)}}^{q} 2^{b q(i-k)}\right\}^{1 / q} \\
& \approx\left\{\sum_{i=-\infty}^{-2} 2^{\alpha_{\infty} i q}\left\|\left(\sum_{j=1}^{\infty}\left|f_{j}\right|^{r}\right)^{1 / r} x_{i}\right\|_{L^{p(\cdot)}}^{q} \sum_{k=0}^{\infty} 2^{b q(i-k)}\right. \\
& \left.+\sum_{i=-1}^{\infty} 2^{\alpha_{\infty} i q}\left\|\left(\sum_{j=1}^{\infty}\left|f_{j}\right|^{r}\right)^{1 / r} x_{i}\right\|_{L^{p(\cdot)}}^{q} \sum_{k=i+2}^{\infty} 2^{b q(i-k)}\right\}^{1 / q} \\
& \lesssim\left\{\sum_{i=-\infty}^{-2} 2^{n \delta_{2} i q}\left\|\left(\sum_{j=1}^{\infty}\left|f_{j}\right|^{r}\right)^{1 / r} x_{i}\right\|_{L^{p()}}^{q}+\sum_{i=-1}^{\infty} 2^{\alpha_{\infty} i q}\left\|\left(\sum_{j=1}^{\infty}\left|f_{j}\right|^{r}\right)^{1 / r} x_{i}\right\|_{L^{p(\cdot)}}^{q}\right\}^{1 / q} \\
& \lesssim\left\{\sum_{i=-\infty}^{-2} 2^{\alpha(0) i q+\left(n \delta_{2}-\alpha(0)\right) i q}\left\|\left(\sum_{j=1}^{\infty}\left|f_{j}\right|^{r}\right)^{1 / r} x_{i}\right\|_{L^{p(\cdot)}}^{q}\right. \\
& \left.+\sum_{i=-1}^{\infty} 2^{\alpha_{\infty} i q}\left\|\left(\sum_{j=1}^{\infty}\left|f_{j}\right|^{r}\right)^{1 / r} x_{i}\right\|_{L^{p(\cdot)}}^{q}\right\}^{1 / q} \\
& \lesssim\left\{\sum_{i=-\infty}^{-2} 2^{\alpha(0) i q}\left\|\left(\sum_{j=1}^{\infty}\left|f_{j}\right|^{r}\right)^{1 / r} x_{i}\right\|_{L^{p())}}^{q}+\sum_{i=-1}^{\infty} 2^{\alpha_{\infty} i q}\left\|\left(\sum_{j=1}^{\infty}\left|f_{j}\right|^{r}\right)^{1 / r} x_{i}\right\|_{L^{p()}}^{q}\right\}^{1 / q} \\
& \lesssim\left\|\left\{2^{\alpha(0) k}\left(\sum_{j=1}^{\infty}\left|f_{j}\right|^{r}\right)^{1 / r} x_{k}\right\}\right\|_{\ell_{<}^{q}\left(L^{p(\cdot)}\right)}+\left\|\left\{2^{\alpha_{\infty} k}\left(\sum_{j=1}^{\infty}\left|f_{j}\right|^{r}\right)^{1 / r} x_{k}\right\}\right\|_{\ell_{>}^{q}\left(L^{p \cdot(\cdot)}\right)} .
\end{aligned}
$$

Thus

$$
I:=I_{<}+I_{>} \lesssim\left\|\left(\sum_{j=1}^{\infty}\left|f_{j}\right|^{r}\right)^{1 / r}\right\|_{\dot{K}_{p(\cdot)}^{\alpha, q}} .
$$


Step 3. We consider III. we have

For for all $i \geq k+2, x \in R_{k}$, by size condition and generalized Minkowski inequality,

$$
\begin{aligned}
\left(\sum_{j=1}^{\infty} M^{r}\left(f_{j}^{i}\right)(x)\right)^{1 / r} & \lesssim\left[\sum_{j=1}^{\infty}\left(2^{-i n} \int_{\mathbb{R}^{n}}\left|f_{j}^{i}(y)\right| d y\right)^{r}\right]^{1 / r} \\
& \approx 2^{-i n}\left(\sum_{j=1}^{\infty}\left(\int_{\mathbb{R}^{n}}\left|f_{j}^{i}(y)\right| d y\right)^{r}\right)^{1 / r} \\
& \lesssim 2^{-i n} \int_{\mathbb{R}^{n}}\left(\sum_{j=1}^{\infty}\left|f_{j}^{i}\right|^{r}\right)^{1 / r} d y .
\end{aligned}
$$

By Hölder's inequality we get

$$
\begin{aligned}
I I I_{<} & \lesssim\left\{\sum_{k=-\infty}^{-1} 2^{\alpha(0) q k}\left\|X_{k} \sum_{i=k+2}^{\infty} 2^{-i n} \int_{\mathbb{R}^{n}}\left(\sum_{j=1}^{\infty}\left|f_{j}^{i}\right|^{r}\right)^{1 / r} d y\right\|_{L^{p(\cdot)}}^{q}\right\}^{1 / q} \\
& \approx\left\{\sum_{k=-\infty}^{-1} 2^{\alpha(0) q k}\left\|X_{k}\right\|_{L^{p(\cdot)}}^{q}\left(\sum_{i=k+2}^{\infty} 2^{-i n} \int_{\mathbb{R}^{n}}\left(\sum_{j=1}^{\infty}\left|f_{j}^{i}\right|^{r}\right)^{1 / r} d y\right)^{q}\right\}^{1 / q} \\
& \lesssim\left\{\sum_{k=-\infty}^{-1} 2^{\alpha(0) q k}\left\|X_{X k}\right\|_{L^{p(\cdot)}}^{q} \times\left(\sum_{i=k+2}^{\infty} 2^{-i n}\left\|\left(\sum_{j=1}^{\infty}\left|f_{j}\right|^{r}\right)^{1 / r}\right\| X_{i}\left\|X_{i}\right\|_{L^{p^{\prime}(\cdot)}}\right)^{q}\right\}^{1 / q} .
\end{aligned}
$$

On the other hand, by using Lemmas 2.2 and 2.3, we have

$$
\begin{aligned}
2^{-i n}\left\|X_{k}\right\|_{L^{p()}}\left\|X_{X_{i}}\right\|_{L^{p^{(\cdot)}}} & \leqslant 2^{-i n}\left\|X_{B_{k}}\right\|_{L^{p()}}\left\|X_{B_{i}}\right\|_{L^{p^{\prime}(\cdot)}} \\
& \lesssim 2^{-i n}\left\|X_{B_{k}}\right\|_{L^{p()}}\left|B_{i}\right|\left\|X_{B_{i}}\right\|_{L^{p(\cdot)}}^{-1} \\
& \lesssim 2^{n \delta_{1}(k-i)} .
\end{aligned}
$$


We put (2.37) into (2.36) and get

$$
\begin{aligned}
I I I_{<} & \lesssim\left\{\sum_{k=-\infty}^{-1} 2^{\alpha(0) k q}\left(\sum_{i=k+2}^{\infty}\left\|\left(\sum_{j=1}^{\infty}\left|f_{j}\right|^{r}\right)^{1 / r} x_{i}\right\|_{L^{p(\cdot)}} 2^{n \delta_{1}(k-i)}\right)^{q}\right\}^{1 / q} \\
& \approx\left\{\sum_{k=-\infty}^{-1}\left(\sum_{i=k+2}^{\infty} 2^{\alpha(0) k}\left\|\left(\sum_{j=1}^{\infty}\left|f_{j}\right|^{r}\right)^{1 / r} x_{i}\right\|_{L^{p(\cdot)}} 2^{n \delta_{1}(k-i)}\right)^{q}\right\}^{1 / q} \\
& \approx\left\{\sum_{k=-\infty}^{-1}\left(\sum_{i=k+2}^{\infty} 2^{\alpha(0) i}\left\|\left(\sum_{j=1}^{\infty}\left|f_{j}\right|^{r}\right)^{1 / r} x_{i}\right\|_{L^{p(\cdot)}} 2^{b(k-i)}\right)^{q}\right\}^{1 / q},
\end{aligned}
$$

here $b=n \delta_{1}+\alpha(0)>0$.

Similar to Step 2, we can make (2.38) further calculation and obtain

$$
I I I_{<} \lesssim\left\|\left\{2^{\alpha(0) k}\left(\sum_{j=1}^{\infty}\left|f_{j}\right|^{r}\right)^{1 / r} \chi_{k}\right\}\right\|_{\ell_{<}^{q}\left(L^{p())}\right)}+\left\|\left\{2^{\alpha_{\infty} k}\left(\sum_{j=1}^{\infty}\left|f_{j}\right|^{r}\right)^{1 / r} x_{k}\right\}\right\|_{\ell_{>}^{q}\left(L^{p(\cdot)}\right)}
$$

Similarly, we have

$$
I I I_{>} \lesssim\left\{\sum_{k=0}^{\infty}\left(\sum_{i=k+2}^{\infty} 2^{\alpha_{\infty} i}\left\|\left(\sum_{j=1}^{\infty}\left|f_{j}\right|^{r}\right)^{1 / r} x_{i}\right\|_{L^{p(\cdot)}} 2^{b(k-i)}\right)^{q}\right\}^{1 / q}
$$

here $b=n \delta_{1}+\alpha_{\infty}>0$. By the same argument followed (2.28), we have

$$
I I I_{>} \lesssim\left\|\left\{2^{\alpha_{\infty} k}\left(\sum_{j=1}^{\infty}\left|f_{j}\right|^{r}\right)^{1 / r} x_{k}\right\}\right\|_{\ell_{>}^{q}\left(L^{p(\cdot)}\right)}
$$

Thus

$$
I I I:=I I I_{<}+I I I_{>} \lesssim\left\|\left(\sum_{j=1}^{\infty}\left|f_{j}\right|^{r}\right)^{1 / r}\right\|_{\hat{K}_{p(),}^{\alpha(), q}}
$$

We get what we want by putting $I, I I$, and $I I I$ into (2.20).

We remark that there is an analogue of Theorem 2.8 for sublinear operators. 
Theorem 2.9. Let $p(\cdot) \in \mathbb{B}\left(\mathbb{R}^{n}\right), 0<q<\infty$ and $\alpha \in L^{\infty}\left(\mathbb{R}^{n}\right)$ be log-Hölder continuous, both at the origin and at infinity, such that $-n \delta_{1}<\alpha(0) \leqslant \alpha_{\infty}<n \delta_{2}$, where $0<\delta_{1}, \delta_{2}<1$ are constants satisfying (2.4), Suppose that $T$ is a sublinear and bounded operator on $L^{p(\cdot)}\left(\mathbb{R}^{n}\right)$ satisfying size condition

$$
|T f(x)| \leqslant C \int_{\mathbb{R}^{n}}|x-y|^{-n}|f(y)| d y,
$$

for all $f \in L_{\text {loc }}^{1}\left(\mathbb{R}^{n}\right)$ with compact support and a.e. $x \notin \operatorname{supp} f$, and for any $1<r<\infty$, there exists a positive constant $C$ such that for all sequences $\left\{f_{j}\right\}_{j=1}^{\infty}$ of locally integrable functions on $\mathbb{R}^{n}$,

$$
\left\|\left(\sum_{j=1}^{\infty}\left|T f_{j}\right|^{r}\right)^{1 / r}\right\|_{L^{p(\cdot)}} \leqslant C\left\|\left(\sum_{j=1}^{\infty}\left|f_{j}\right|^{r}\right)^{1 / r}\right\|_{L^{p(\cdot)}} .
$$

Then

$$
\begin{aligned}
& \left\|\left(\sum_{k=1}^{\infty}\left|T f_{k}\right|^{r}\right)^{1 / r}\right\|_{\dot{K}_{p(\cdot)}^{\alpha(\cdot), q}\left(\mathbb{R}^{n}\right)} \leqslant C\left\|\left(\sum_{k=1}^{\infty}\left|f_{k}\right|^{r}\right)^{1 / r}\right\|_{\dot{K}_{p(\cdot)}^{\alpha(\cdot), q}\left(\mathbb{R}^{n)}\right.}, \\
& \left\|\left(\sum_{k=1}^{\infty}\left|T f_{k}\right|^{r}\right)^{1 / r}\right\|_{K_{p(\cdot)}^{\alpha(\cdot), q}\left(\mathbb{R}^{n}\right)} \leqslant C\left\|\left(\sum_{k=1}^{\infty}\left|f_{k}\right|^{r}\right)^{1 / r}\right\|_{K_{p(\cdot)}^{\alpha(\cdot), q}\left(\mathbb{R}^{n}\right)},
\end{aligned}
$$

for all sequences $\left\{f_{j}\right\}_{j=1}^{\infty}$ of locally integrable functions on $\mathbb{R}^{n}$, where $C$ is independent of $\left\{f_{j}\right\}_{j=1}^{\infty}$.

\section{The Quasi-Norm Characterizations}

Let $\mathcal{S}\left(\mathbb{R}^{n}\right)$ be the Schwartz space on $\mathbb{R}^{n}, \mathcal{S}^{\prime}\left(\mathbb{R}^{n}\right)$ be its dual space on $\mathbb{R}^{n}$. For $\varphi \in \mathcal{S}\left(\mathbb{R}^{n}\right), \widehat{\varphi}$ denotes its Fourier transform, and $\varphi^{\vee}$ denotes its inverse Fourier transform. Take $\varphi_{0} \in \mathcal{S}\left(\mathbb{R}^{n}\right)$ with $\varphi_{0}(x) \geq 0$ and

$$
\varphi_{0}(x)= \begin{cases}1, & |x| \leqslant 1 \\ 0, & |x| \geq 2\end{cases}
$$

Now define $\varphi(x)=\varphi_{0}(x)-\varphi_{0}(2 x)$ and set $\varphi_{j}(x)=\varphi\left(2^{-j} x\right)$ for all $j \in \mathbb{N}$. Let $\mathbb{N}_{0}=\mathbb{N} \cup\{0\}$. Then $\left\{\varphi_{j}\right\}_{j \in \mathbb{N}_{0}}$ is a resolution of unity, that means $\sum_{j=0}^{\infty} \varphi_{j}(x)=1$ for all $x \in \mathbb{R}^{n}$.

Now, we introduce the Herz-type Besov spaces and Triebel-Lizorkin spaces with variable exponents as following.

Definition 3.1. Let $\left\{\varphi_{j}\right\}_{j \in \mathbb{N}_{0}}$ be a resolution of unity as above, $s \in \mathbb{R}, 0<\beta, q \leq \infty, p(\cdot) \in p^{0}\left(\mathbb{R}^{n}\right)$ and $\alpha(\cdot): \mathbb{R}^{n} \rightarrow \mathbb{R}$ with $\alpha(\cdot) \in L^{\infty}\left(\mathbb{R}^{n}\right)$. 
(i) The set

$$
\left\{f \in \mathcal{S}^{\prime}\left(\mathbb{R}^{n}\right):\left\|\left\{2^{s j} \varphi_{j}^{\vee} * f\right\}_{j=0}^{\infty}\right\|_{\mathcal{R}_{\beta}\left(K_{p(\cdot)}^{\alpha(), q)}\right)}<\infty\right\}
$$

is named to the Herz-type Besov space with variable exponents and denoted by $K_{p(\cdot)}^{\alpha(\cdot), q} B_{\beta}^{s}\left(\mathbb{R}^{n}\right)$. The quasi-norm of $f$ in this space is denoted by

$$
\|f\|_{K_{p(\cdot)}^{\alpha(\cdot), q} B_{\beta}^{s}\left(\mathbb{R}^{n}\right)}:=\left\|\left\{2^{s j} \varphi_{j}^{\vee} * f\right\}_{j=0}^{\infty}\right\|_{\ell_{\beta}\left(K_{p(\cdot)}^{\alpha(\cdot), q}\right)}
$$

(ii) For $p^{+}<\infty$, the set

$$
\left\{f \in \mathcal{S}^{\prime}\left(\mathbb{R}^{n}\right):\left\|\left\{2^{s j} \varphi_{j}^{\vee} * f\right\}_{j=0}^{\infty}\right\|_{K_{p(\cdot)}^{\alpha(\cdot)}\left(\ell_{\beta}\right)}<\infty\right\}
$$

is named to the Herz-type Triebel-Lizorkin space with variable exponents and denoted by $K_{p(\cdot)}^{\alpha(\cdot), q} F_{\beta}^{s}\left(\mathbb{R}^{n}\right)$. The quasi-norm of $f$ in this space is denoted by

$$
\|f\|_{K_{p(\cdot)}^{\alpha(\cdot), q} F_{\beta}^{s}\left(\mathbb{R}^{n}\right)}:=\left\|\left\{2^{s j} \varphi_{j}^{\vee} * f\right\}_{j=0}^{\infty}\right\|_{K_{p(\cdot)}^{\alpha(\cdot), q}\left(e_{\beta}\right)} .
$$

Here $\ell_{\beta}\left(K_{p(\cdot)}^{\alpha(\cdot), q}\right)$ and $K_{p(\cdot)}^{\alpha(\cdot), q}\left(\ell_{\beta}\right)$ are the spaces of all sequences $\left\{g_{j}\right\}$ of measurable functions on $\mathbb{R}^{n}$ with finite quasi-norms

$$
\begin{gathered}
\left\|\left\{g_{j}\right\}\right\|_{\ell_{\beta}\left(K_{p(\cdot)}^{\alpha(\cdot), q}\right)}:=\left(\sum_{j=0}^{\infty}\left\|g_{j}\right\|_{K_{p())}^{\alpha(\cdot), q}}^{\beta}\right)^{1 / \beta}, \\
\left\|\left\{g_{j}\right\}_{j=0}^{\infty}\right\|_{K_{p(\cdot)}^{\alpha(\cdot),\left(\ell_{\beta}\right)}}:=\left\|\left(\sum_{j=0}^{\infty}\left|g_{j}\right|^{\beta}\right)^{1 / \beta}\right\|_{K_{p(\cdot)}^{\alpha(\cdot), q}} .
\end{gathered}
$$

The spaces $\dot{K}_{p(\cdot)}^{\alpha(\cdot), q} B_{\beta}^{s}\left(\mathbb{R}^{n}\right)$ and $\dot{K}_{p(\cdot)}^{\alpha(\cdot), q} F_{\beta}^{s}\left(\mathbb{R}^{n}\right)$ are defined similarly by replacing the norm $K_{p(\cdot)}^{\alpha(\cdot), q}$ with $\dot{K}_{p(\cdot)}^{\alpha(\cdot), q}$. To make these spaces definite, we need to show them independent of the choice of the resolution of unity $\left\{\varphi_{j}\right\}_{j \in \mathbb{N}_{0}}$. To this aim we need more notation. 
Let $\Psi_{0}, \Psi \in \mathcal{S}\left(\mathbb{R}^{n}\right), \epsilon>0$, integer $S \geq-1$ be such that

$$
\begin{gathered}
\left|\widehat{\Psi_{0}}(\xi)\right|>0 \quad \text { on }\{|\xi|<2 \varepsilon\}, \\
|\widehat{\Psi}(\xi)|>0 \quad \text { on }\left\{\frac{\varepsilon}{2}<|\xi|<2 \varepsilon\right\}, \\
D^{\tau} \widehat{\Psi}(0)=0 \quad \forall|\tau| \leqslant S .
\end{gathered}
$$

Here (3.7) and (3.8) are Tauberian conditions, while (3.9) expresses moment conditions on $\Psi$.

Let us recall the classical Peetre maximal operator introduced in [34]. Given a sequence of function $\left\{\Psi_{k}\right\}_{k \in \mathbb{Z}} \subset \mathcal{S}\left(\mathbb{R}^{n}\right)$, a tempered distribution $f \in \mathcal{S}^{\prime}\left(\mathbb{R}^{n}\right)$ and a positive number $a>0$, we define the system of maximal functions

$$
\left(\Psi_{k}^{*}\right)_{a} f(x):=\sup _{y \in \mathbb{R}^{n}} \frac{\left|\Psi_{k} * f(x+y)\right|}{\left(1+2^{k}|y|\right)^{a}}, \quad x \in \mathbb{R}^{n}, k \in \mathbb{Z} .
$$

Since $\Psi_{k} * f(y)$ makes sense pointwise everything is well defined. We will often use dilates $\Psi_{k}(x)=2^{k n} \Psi\left(2^{k} x\right)$ of a fixed function $\Psi \in \mathcal{S}\left(\mathbb{R}^{n}\right)$, where $\Psi_{0}(x)$ might be given by a separate function. Also continuous dilates are needed. Let $\Psi_{t}(\cdot):=t^{-n} \Psi\left(t^{-1} \cdot\right)$. We define $\left(\Psi_{t}^{*}\right)_{a} f(x)$ by

$$
\left(\Psi_{t}^{*}\right)_{a} f(x):=\sup _{y \in \mathbb{R}^{n}} \frac{\left|\Psi_{t} * f(x+y)\right|}{(1+|y| / t)^{a}} \quad x \in \mathbb{R}^{n}, t>0
$$

Now we have equivalent quasi-norms for these new spaces.

Theorem 3.2. Let $s<S+1,0<\beta, q \leqslant \infty, p(\cdot) \in p^{0}\left(\mathbb{R}^{n}\right)$ for $p(\cdot) / p_{0} \in \mathbb{B}\left(\mathbb{R}^{n}\right)$ with $p_{0}<$ $\min \left(p_{-}, 1\right), \alpha(\cdot): \mathbb{R}^{n} \rightarrow \mathbb{R}$ with $\alpha(\cdot) \in L^{\infty}\left(\mathbb{R}^{n}\right)$ and $\alpha(\cdot)$ be log-Hölder continuous, both at the origin and at infinity, such that $-n \delta_{1}<\alpha(0) p_{0} \leqslant \alpha_{\infty} p_{0}<n \delta_{2}$, where $0<\delta_{1}, \delta_{2}<1$ are constants satisfying (2.4) for $p(\cdot) / p_{0}$. And let $a \in \mathbb{R}$. Further, let $\Phi_{0}, \Phi$ belong to $\mathcal{S}\left(\mathbb{R}^{n}\right)$ be given by (3.7), (3.8), and (3.9).

(i) For $a>n / p_{0}$, then the space $K_{p(\cdot)}^{\alpha(\cdot), q} B_{\beta}^{s}$ can be characterized by

$$
K_{p(\cdot)}^{\alpha(\cdot), q} B_{\beta}^{s}\left(\mathbb{R}^{n}\right)=\left\{f \in \mathcal{S}^{\prime}\left(\mathbb{R}^{n}\right):\|f\|_{K_{p(\cdot)}^{\alpha(\cdot), q} B_{\beta}^{s}\left(\mathbb{R}^{n}\right)}^{(i)}<\infty\right\}, \quad i=1, \ldots, 4
$$


where

$$
\begin{aligned}
& \|f\|_{K_{p(\cdot)}^{\alpha(\cdot), q} B_{\beta}^{s}\left(\mathbb{R}^{n}\right)}^{(1)}:=\left\|\Phi_{0} * f\right\|_{K_{p(\cdot)}^{\alpha(\cdot), q}}+\left(\int_{0}^{1} t^{-s \beta}\left\|\left(\Phi_{t} * f\right)(\cdot)\right\|_{K_{p(\cdot)}^{\alpha(\cdot), q}\left(\mathbb{R}^{n}\right)}^{\beta} \frac{d t}{t}\right)^{1 / \beta}, \\
& \|f\|_{K_{p(\cdot)}^{\alpha(\cdot), q} B_{\beta}^{s}\left(\mathbb{R}^{n}\right)}^{(2)}:=\left\|\left(\Phi_{0}^{*}\right)_{a} f\right\|_{K_{p(\cdot)}^{\alpha(\cdot), q}}+\left(\int_{0}^{1} t^{-s \beta}\left\|\left(\Phi_{t}^{*}\right)_{a} f\right\|_{K_{p(\cdot)}^{\alpha(\cdot),\left(\mathbb{R}^{n}\right)}}^{\beta} \frac{d t}{t}\right)^{1 / \beta}, \\
& \|f\|_{K_{p(\cdot)}^{\alpha(\cdot), q} B_{\beta}^{s}\left(\mathbb{R}^{n}\right)}^{(3)}:=\left(\sum_{k=0}^{\infty} 2^{s k \beta}\left\|\left(\Phi_{k}^{*}\right)_{a} f\right\|_{K_{p(\cdot)}^{\alpha(\cdot), q}\left(\mathbb{R}^{n}\right)}^{\beta}\right)^{1 / \beta}, \\
& \|f\|_{K_{p(\cdot)}^{\alpha(\cdot), q} B_{\beta}^{s}\left(\mathbb{R}^{n}\right)}^{(4)}:=\left(\sum_{k=0}^{\infty} 2^{s k \beta}\left\|\Phi_{k} * f\right\|_{K_{p(\cdot)}^{\alpha(\cdot), q}\left(\mathbb{R}^{n}\right)}^{\beta}\right)^{1 / \beta} .
\end{aligned}
$$

Furthermore, $\left\{\|\cdot\|_{K_{p(\cdot)}^{\alpha(\cdot), q} B_{\beta}^{s}\left(\mathbb{R}^{n}\right)}^{(i)}\right\}_{i=1}^{4}$ are equivalent.

(ii) If $p_{0}<\beta$, then for $a>n / p_{0}$ the space $K_{p(\cdot)}^{\alpha(\cdot), q} F_{\beta}^{s}$ can be characterized by

$$
K_{p(\cdot)}^{\alpha(\cdot), q} F_{\beta}^{s}\left(\mathbb{R}^{n}\right)=\left\{f \in \mathcal{S}^{\prime}\left(\mathbb{R}^{n}\right):\|f\|_{K_{p(\cdot)}^{\alpha(\cdot), q} F_{\beta}^{s}\left(\mathbb{R}^{n}\right)}^{(i)}<\infty\right\}, \quad i=1, \ldots, 5,
$$

where

$$
\begin{aligned}
& \|f\|_{K_{p(\cdot)}^{\alpha(\cdot), q} F_{\beta}^{s}\left(\mathbb{R}^{n}\right)}^{(1)}:=\left\|\Phi_{0} * f\right\|_{K_{p(\cdot)}^{\alpha(\cdot), q}}+\left\|\left(\int_{0}^{1} t^{-s \beta}\left|\Phi_{t} * f(\cdot)\right|^{\beta} \frac{d t}{t}\right)^{1 / \beta}\right\|_{K_{p(\cdot)}^{\alpha(\cdot), q\left(\mathbb{R}^{n}\right)}}, \\
& \|f\|_{K_{p(\cdot)}^{\alpha(\cdot), q} F_{\beta}^{s}\left(\mathbb{R}^{n}\right)}^{(2)}:=\left\|\left(\Phi_{0}^{*}\right)_{a} f\right\|_{K_{p(\cdot)}^{\alpha(\cdot), q}}+\left\|\left(\int_{0}^{1}\left[t^{-s}\left(\Phi_{t}^{*}\right)_{a} f\right]^{\beta} \frac{d t}{t}\right)^{1 / \beta}\right\|_{K_{p(\cdot)}^{\alpha(\cdot), q\left(\mathbb{R}^{n}\right)}}, \\
& \|f\|_{K_{p(\cdot)}^{\alpha(\cdot),} F_{\beta}^{s}\left(\mathbb{R}^{n)}\right.}^{(3)}:=\left\|\Phi_{0} * f\right\|_{K_{p(\cdot)}^{\alpha(\cdot), q}\left(\mathbb{R}^{n}\right)}+\left\|\left(\int_{0}^{1} t^{-s \beta} \int_{|z|<t}\left|\left(\Phi_{t} * f\right)(\cdot+z)\right|^{\beta} d z \frac{d t}{t^{n+1}}\right)^{1 / \beta}\right\|_{K_{p(\cdot)}^{\alpha(\cdot), q\left(\mathbb{R}^{n}\right)}}, \\
& \|f\|_{K_{p(\cdot)}^{\alpha(\cdot), q} F_{\beta}^{s}\left(\mathbb{R}^{n}\right)}^{(4)}:=\left\|\left(\sum_{k=0}^{\infty}\left[2^{k s \beta}\left(\Phi_{k}^{*}\right)_{a} f\right]^{\beta}\right)^{1 / \beta}\right\|_{K_{p(\cdot)}^{\alpha(\cdot), q}\left(\mathbb{R}^{n}\right)}, \\
& \|f\|_{K_{p(\cdot)}^{\alpha(\cdot), q} F_{\beta}^{s}\left(\mathbb{R}^{n}\right)}^{(5)}:=\left\|\left(\sum_{k=0}^{\infty} 2^{k s \beta}\left|\Phi_{k} * f\right|^{\beta}\right)^{1 / \beta}\right\|_{K_{p(\cdot)}^{\alpha(\cdot), q}} .
\end{aligned}
$$

Furthermore, $\left\{\|\cdot\|_{K_{p(\cdot)}^{\alpha(\cdot), q} F_{\beta}^{s}\left(\mathbb{R}^{n}\right)}^{(i)}\right\}_{i=1}^{5}$ are equivalent. 
Theorem 3.2 is the generalization of Theorem 1 in [28] for the Herz type space $K_{p(\cdot)}^{\alpha, q}$ only one variable exponent $p(\cdot)$. For readers' convenience, we give the proof for $F$-parts in outline. The proof of $B$-parts is similar and a bit simpler. The idea of the proof goes back to [35]. In fact, we will use the argument in [35]. To go on, we need some lemmas.

Lemma 3.3 (see [36]). Let $\mu, v \in \mathcal{S}\left(\mathbb{R}^{n}\right),-1 \leqslant M \in \mathbb{Z}$,

$$
D^{\tau} \widehat{\mu}(0)=0 \quad \forall|\tau| \leqslant M .
$$

Then for any $N>0$ there is a constant $C_{N}$ so that

$$
\sup _{z \in \mathbb{R}^{n}}\left|\mu_{t} * \mathcal{v}(z)\right|(1+|z|)^{N} \leqslant C_{N} t^{M+1}
$$

where $\mu_{t}(x)=t^{-n} \mu(x / t)$ for all $0<t \leqslant 2$.

Lemma 3.4 (see [36]). Let $0<q \leqslant \infty, \delta>0$. For any sequence $\left\{g_{j}\right\}_{0}^{\infty}$ of nonnegative numbers denote

$$
G_{j}=\sum_{k=0}^{\infty} 2^{-|k-j| \delta} g_{k}
$$

Then

$$
\left\|\left\{G_{j}\right\}_{0}^{\infty}\right\|_{\ell_{q}} \leqslant C\left\|\left\{g_{j}\right\}_{0}^{\infty}\right\|_{\ell_{q^{\prime}}}
$$

holds, where $C$ is constant only depend on $q, \delta$.

Lemma 3.5. Let $0<\beta \leqslant \infty, \delta>0,0<q \leqslant \infty, p(\cdot) \in p^{0}\left(\mathbb{R}^{n}\right)$ and $\alpha(\cdot): \mathbb{R}^{n} \rightarrow \mathbb{R}$ with $\alpha(\cdot) \in L^{\infty}\left(\mathbb{R}^{n}\right)$. For any sequence $\left\{g_{j}\right\}_{0}^{\infty}$ of nonnegative measurable functions on $\mathbb{R}^{n}$ denote

$$
G_{j}(x)=\sum_{k=0}^{\infty} 2^{-|k-j| \delta} g_{k}(x), \quad x \in \mathbb{R}^{n}
$$

Then

$$
\begin{aligned}
& \left\|\left\{G_{j}\right\}_{0}^{\infty}\right\|_{K_{p(\cdot)}^{\alpha(\cdot), q}\left(\ell_{\beta}\right)} \leqslant C_{1}\left\|\left\{g_{j}\right\}_{0}^{\infty}\right\|_{K_{p(\cdot)}^{\alpha(\cdot), q}\left(\ell_{\beta}\right)^{\prime}} \\
& \left\|\left\{G_{j}\right\}_{0}^{\infty}\right\|_{\ell_{\beta}\left(K_{p(\cdot)}^{\alpha(\cdot), q}\right)} \leqslant C_{2}\left\|\left\{g_{j}\right\}_{0}^{\infty}\right\|_{\ell_{\beta}\left(K_{p(\cdot)}^{\alpha(\cdot), q}\right)^{\prime}}
\end{aligned}
$$

hold with some constants $C_{1}=C_{1}(q, \delta)$ and $C_{2}=C_{2}(p(\cdot), q, \delta)$.

Proof. By Lemma 3.4, (3.25) follows immediately from (3.23). Now we prove (3.26). Let $p(\cdot) \in D^{0}\left(\mathbb{R}^{n}\right)$. Here we can divide it into two cases. 
Case $1\left(p_{-} \geq 1, q \geq 1\right)$. Since $\|\cdot\|_{K_{p(\cdot)}^{\alpha(\cdot), q}}$ is a norm, we have

$$
\left\|G_{j}\right\|_{K_{p(\cdot)}^{\alpha(\cdot), q}} \leqslant \sum_{k=0}^{\infty} 2^{-|k-j| \delta}\left\|g_{k}\right\|_{K_{p(\cdot)}^{\alpha(\cdot), q}}
$$

By Lemma 3.4, we obtain (3.26).

Case 2. If $q<1$, take $p_{0}<\min \left(p_{-}, q\right)$. Then

$$
\begin{aligned}
\left\|G_{j}\right\|_{K_{p(\cdot)}^{\alpha(\cdot), q}}^{p_{0}} & =\left\|\left|G_{j}\right|^{p_{0}}\right\|_{K_{p(\cdot) / p_{0}}^{\alpha(\cdot) p_{0}, q / p_{0}}} \\
& \leqslant\left\|\sum_{k=0}^{\infty} 2^{-|k-j| p_{0} \delta}\left|g_{k}\right|^{p_{0}}\right\|_{K_{p(\cdot) / p_{0}}^{\alpha(\cdot) / p_{0}, q / p_{0}}} \\
& \leqslant \sum_{k=0}^{\infty} 2^{-|k-j| p_{0} \delta}\left\|\left|g_{k}\right|^{p_{0}}\right\|_{K_{p(\cdot) / p_{0}}^{\alpha(\cdot), q, q / p_{0}}} .
\end{aligned}
$$

By Lemma 3.4, we obtain

$$
\begin{aligned}
\left\|\left\{G_{j}\right\}\right\|_{\ell_{\beta}\left(K_{p(\cdot)}^{\alpha(\cdot), q}\right)}^{p_{0}} & =\left\|\left\{\left|G_{j}\right|^{p_{0}}\right\}\right\|_{\ell_{\beta / p_{0}}\left(K_{p(\cdot) / p_{0}}^{\alpha(\cdot) p_{0}, q / p_{0}}\right)} \\
& \lesssim\left\|\left\{\left|g_{k}\right|^{p_{0}}\right\}\right\|_{\ell_{\beta / p_{0}}\left(K_{p(\cdot) / p_{0}}^{\alpha(\cdot), p_{0}, q / p_{0}}\right)} \\
& =C\left\|\left\{g_{k}\right\}\right\|_{\ell_{\beta}\left(K_{p(\cdot)}^{\alpha(\cdot), q}\right)}^{p_{0}} .
\end{aligned}
$$

In the last inequality, we used (3.26) has been proved for space $K_{p(\cdot) / p_{0}}^{\alpha(\cdot) p_{0} q / p_{0}}$. Raising to power $1 / p_{0}$, we obtain (3.26).

Lemma 3.6 (Theorem 6 in [37]). Let $\left\{\varphi_{j}\right\}_{j \in \mathbb{N}_{0}}$ is a resolution of unity and let $R \in \mathbb{N}$. Then there exist functions $\theta_{0}, \theta \in \mathcal{S}\left(\mathbb{R}^{n}\right)$ satisfy

$$
\begin{gathered}
\operatorname{supp} \theta_{0}, \quad \operatorname{supp} \theta \subseteq\left\{x \in \mathbb{R}^{n}:|x| \leqslant 1\right\}, \\
\left|\hat{\theta}_{0}(\xi)\right|>0 \quad \text { on }\{|\xi|<2 \epsilon\}, \\
|\widehat{\theta}(\xi)|>0 \text { on }\left\{\frac{\epsilon}{2}<|\xi|<2 \epsilon\right\}, \\
\int_{\mathbb{R}^{n}} x^{r} \theta(x) d x=0 \quad \text { for } 0<|\gamma| \leqslant R
\end{gathered}
$$

such that

$$
\widehat{\theta_{0}}(\xi) \widehat{\psi_{0}}(\xi)+\sum_{j=1}^{\infty} \widehat{\theta}\left(2^{-j} \xi\right) \widehat{\psi}\left(2^{-j} \xi\right)=1, \quad \forall \xi \in \mathbb{R}^{n}
$$

where the functions $\psi_{0}, \psi \in \mathcal{S}\left(\mathbb{R}^{n}\right)$ are defined via $\widehat{\psi_{0}}(\xi)=\varphi_{0}(\xi) / \widehat{\theta_{0}}(\xi)$ and $\widehat{\psi}(\xi)=\varphi_{1}(2 \xi) / \widehat{\theta}(\xi)$. 
Proof of Theorem 3.2. We divide the total proof into four steps. First, we prove the equivalence of (3.15) and (3.16). The next step is to build the bridge between (3.16) and (3.18) and to change from the system $\left(\Phi_{0}, \Phi\right)$ to a system $\left(\Psi_{0}, \Psi\right)$. The equivalence of (3.18) and (3.19) goes parallel to (3.15) and (3.16). Indeed, Definition 3.1 can be seen as a special case of (3.19). Finally, we prove (3.19) is equivalent to the rest.

Step 1 . We are going to prove that for every $f \in S^{\prime}\left(\mathbb{R}^{n}\right)$

$$
\|f\|_{K_{p(\cdot)}^{\alpha(\cdot), q} F_{\beta}^{s}}^{(2)} \lesssim\|f\|_{K_{p(\cdot)}^{\alpha(\cdot), q} F_{\beta}^{s}}^{(1)} \lesssim\|f\|_{K_{p(\cdot)}^{\alpha(\cdot), q} F_{\beta}^{s}}^{(2)}
$$

From Lemmas 3.3 and 3.6, we have that, see [35], for $r<\min \left\{p_{-}, \beta\right\}, N \in \mathbb{N}$, there exists positive constant $C$ for $f \in \mathcal{S}\left(\mathbb{R}^{n}\right)$,

$$
\begin{aligned}
\left(\int_{1}^{2}\left|2^{l s}\left(\Phi_{2^{-l} t}^{*} f\right)_{a}(x)\right|^{\beta} \frac{d t}{t}\right)^{r / \beta} \leqslant & C \sum_{k \in l+\mathbb{N}_{0}} 2^{(l-k)(N r-n+r s)} 2^{k r s} \\
& \times \mathcal{M}\left[\left(\int_{1}^{2}\left|\left(\left(\Phi_{k}\right)_{t} * f\right)(\cdot)\right|^{\beta} \frac{d t}{t}\right)^{r / \beta}\right](x) .
\end{aligned}
$$

Take now $n / a<r=p_{0}<\min \left\{p_{-}, \beta\right\}, N>\max \{0,-s\}+a$ and put $\delta=N+s-d / r>0$. We obtain for $l \in \mathbb{N}$

$$
\begin{aligned}
& \left(\int_{1}^{2}\left|2^{l s}\left(\Phi_{2^{-l} t}^{*} f\right)_{a}(x)\right|^{\beta} \frac{d t}{t}\right)^{r / \beta} \\
& \quad \lesssim \sum_{k \in l+\mathbb{N}_{0}} 2^{-\delta r|l-k|} 2^{k r s} \mathcal{M}\left[\left(\int_{1}^{2}\left|\left(\left(\Phi_{k}\right)_{t} * f\right)(\cdot)\right|^{\beta} \frac{d t}{t}\right)^{r / \beta}\right](x) .
\end{aligned}
$$

Now we apply Lemma 3.5 in $K_{p(\cdot) / r}^{r \alpha(\cdot), r}\left(\ell_{\beta / r}\right)$ which yields

$$
\begin{aligned}
& \left\|\left\{\left(\int_{1}^{2}\left|2^{l s}\left(\Phi_{2^{-l} t}^{*} f\right)_{a}(x)\right|^{\beta} \frac{d t}{t}\right)^{r / \beta}\right\}_{l \in \mathbb{N}}\right\|_{K_{p(\cdot) / r}^{r \alpha(\cdot) / r}\left(\ell_{\beta / r}\right)} \\
& \quad \lesssim\left\|\left\{\mathcal{M}\left[\left(\int_{1}^{2}\left|2^{k s}\left(\left(\Phi_{k}\right)_{t} * f\right)(\cdot)\right|^{\beta} \frac{d t}{t}\right)^{r / \beta}\right]\right\}_{l \in \mathbb{N}}\right\|_{K_{p(\cdot) / r}^{r \alpha(\cdot), q / r}\left(\ell_{\beta / r}\right)} .
\end{aligned}
$$


Next, using Theorem 2.8, we can obtain

$$
\begin{aligned}
& \left\|\left\{\left(\int_{1}^{2}\left|2^{l s}\left(\Phi_{2^{-l} t}^{*} f\right)_{a}(x)\right|^{\beta} \frac{d t}{t}\right)^{r / \beta}\right\}\right\|_{l \in \mathbb{N}} \|_{K_{p(\cdot) / r}^{r \alpha(\cdot), r}\left(e_{\beta / r}\right)} \\
& \lesssim\left\|\left\{\mathcal{M}\left[\left(\int_{1}^{2}\left|2^{k s}\left(\left(\Phi_{k}\right)_{t} * f\right)(\cdot)\right|^{\beta} \frac{d t}{t}\right)^{r / \beta}\right]\right\}_{l \in \mathbb{N}}\right\|_{K_{p(\cdot) / r}^{r \alpha(\cdot) / r}\left(e_{\beta / r}\right)} \\
& \lesssim\left\|\left\{\left(\int_{1}^{2}\left|2^{k s}\left(\left(\Phi_{k}\right)_{t} * f\right)(\cdot)\right|^{\beta} \frac{d t}{t}\right)^{r / \beta}\right\}_{k \in \mathbb{N}}\right\|_{K_{p(\cdot) / r}^{r \alpha(\cdot) / r}\left(\ell_{\beta / r}\right)} \\
& =\left\|\left\{\left(\int_{1}^{2}\left|2^{k s}\left(\left(\Phi_{k}\right)_{t} * f\right)(\cdot)\right|^{\beta} \frac{d t}{t}\right)^{1 / \beta}\right\}_{k \in \mathbb{N}}\right\|_{K_{p(\cdot)}^{\alpha(\cdot), q}\left(\ell_{\beta}\right)}^{r} .
\end{aligned}
$$

Hence, we obtain

$$
\begin{aligned}
\left\|\left(\int_{0}^{1}\left|\lambda^{-s}\left(\Phi_{\lambda}^{*} f\right)_{a}(\cdot)\right|^{\beta} \frac{d \lambda}{\lambda}\right)^{1 / \beta}\right\|_{K_{p(\cdot)}^{\alpha(\cdot), q}} & \approx\left\|\left(\sum_{l=1}^{\infty} \int_{1}^{2}\left|2^{l s}\left(\Phi_{2^{-l} t}^{*} f\right)_{a}(\cdot)\right|^{\beta} \frac{d t}{t}\right)^{1 / \beta}\right\|_{K_{p(\cdot),}^{\alpha(\cdot), q}} \\
& \lesssim\left\|\left\{\left(\int_{1}^{2}\left|2^{l s} \Phi_{2^{-l} t} * f(\cdot)\right|^{\beta} \frac{d t}{t}\right)^{1 / \beta}\right\}_{l \in \mathbb{N}}\right\|_{K_{p(\cdot)}^{\alpha(\cdot), q}\left(e_{\beta}\right)} \\
& \sim\left\|\left(\int_{0}^{1}\left|\lambda^{-s} \Phi_{\lambda} * f(\cdot)\right|^{\beta} \frac{d \lambda}{\lambda}\right)^{1 / \beta}\right\|_{K_{p(\cdot)}^{\alpha(\cdot), q}} .
\end{aligned}
$$

This proves $\|f\|_{K_{p()}^{\alpha(\cdot), q} F_{\beta}^{s}}^{(2)} \lesssim\|f\|_{K_{p())}^{\alpha(\cdot)} F_{\beta}^{s}}^{(1)}$. Since the reverse inequality is trivial, this finishes Step 1.

Step 2. Let $\Psi_{0}, \Psi \in \mathcal{S}^{\prime}\left(\mathbb{R}^{n}\right)$ be functions satisfying (3.25).

First, we are going to prove for all $f \in \mathcal{S}^{\prime}\left(\mathbb{R}^{n}\right)$

$$
\|f\|_{K_{p(\cdot)}^{\alpha(\cdot), q} F_{\beta}^{s}\left(\mathbb{R}^{n}, \Psi\right)}^{(4)} \lesssim\|f\|_{K_{p(\cdot)}^{\alpha(\cdot), q} F_{\beta}^{s}\left(\mathbb{R}^{n}, \Phi\right)}^{(2)} .
$$

Again from Lemmas 3.6 and 3.3, we have that, see [35], let $\delta=\min \{1, S+1-s\}$, there exists a positive constant $C$ for any $f \in \mathcal{S}\left(\mathbb{R}^{n}\right)$,

$$
2^{l s}\left(\Psi_{l}^{*} f\right)_{a}(x) \leqslant C \sum_{k \in \mathbb{N}_{0}} 2^{-|k-l| \delta} 2^{k s}\left(\Phi_{2^{-k} t}^{*} f\right)_{a}(x),
$$

for all $x \in \mathbb{R}^{n}$ and all $t \in[1,2]$. 
Journal of Function Spaces and Applications

Suppose first that $\beta \geq 1$. Then we take on both sides $\left(\int_{1}^{2}|\cdot|^{\beta} d t / t\right)^{1 / \beta}$, which gives

$$
2^{l s}\left(\Psi_{l}^{*} f\right)_{a}(x) \lesssim \sum_{k \in \mathbb{N}_{0}} 2^{-|k-l| \delta} 2^{k s}\left(\int_{1}^{2}\left|\left(\Phi_{2^{-k} t}^{*} f\right)_{a}(x)\right|^{\beta} \frac{d t}{t}\right)^{1 / \beta}
$$

Applying Lemma 3.5 we obtain that

$$
\left\|\left\{2^{l s}\left(\Psi_{l}^{*} f\right)_{a}\right\}_{l \in \mathbb{N}}\right\|_{K_{p(\cdot)}^{\alpha(\cdot), q}\left(e_{\beta}\right)} \lesssim\left\|\left(\sum_{k=1}^{\infty} 2^{k s \beta}\left|\left(\Phi_{2^{-k t}}^{*} f\right)_{a}(x)\right|^{\beta} \frac{d t}{t}\right)^{1 / \beta}\right\|_{K_{p(\cdot)}^{\alpha(\cdot), q}},
$$

which gives the desired result.

In case $\beta<1$ we argue as follows. The quantity $\left(\int_{1}^{2}|\cdot| \beta d t / t\right)^{1 / \beta}$ is no longer a norm. This gives

$$
\left(2^{l s}\left(\Psi_{l}^{*} f\right)_{a}(x)\right)^{\beta} \lesssim \sum_{k \in \mathbb{N}_{0}} 2^{-\beta|k-l| \delta} 2^{k s \beta} \int_{1}^{2}\left|\left(\Phi_{2^{-k} t}^{*} f\right)_{a}(x)\right|^{\beta} \frac{d t}{t}
$$

Notice that the right-hand side is nothing more than a convolution $(\gamma * \alpha(\cdot))_{\ell}$ of the sequences

$$
\gamma_{k}=2^{-|k| \delta \beta}, \quad \alpha(\cdot)_{k}=2^{k s \beta} \int_{1}^{2}\left|\left(\Phi_{2^{-k} t}^{*} f\right)_{a}(x)\right|^{\beta} \frac{d t}{t} .
$$

Now we apply the $\ell_{1}$-norm to both sides and get for all $x \in \mathbb{R}^{n}$

$$
\begin{aligned}
\left\|2^{l s}\left(\Psi_{l}^{*} f\right)_{a}(x)\right\|_{\ell_{\beta}}^{\beta} & \leqslant\|r\|_{\ell_{1}} \cdot\|\alpha(\cdot)\|_{\ell_{1}} \\
& \lesssim \sum_{k=1}^{\infty} 2^{k s \beta} \int_{1}^{2}\left|\left(\Phi_{2^{-k} t}^{*} f\right)_{a}(x)\right|^{\beta} \frac{d t}{t} .
\end{aligned}
$$

We take both sides to power $(\cdots)^{1 / \beta}$ and apply the $K_{p(\cdot)}^{\alpha(\cdot), q}\left(\mathbb{R}^{n}\right)$-norm. This gives (3.38). Similarly, we obtain for all $f \in \mathcal{S}^{\prime}\left(\mathbb{R}^{n}\right)$

$$
\|f\|_{K_{p(\cdot)}^{\alpha(\cdot), q} F_{\beta}^{s}\left(\mathbb{R}^{n}, \Phi\right)}^{(2)} \lesssim\|f\|_{K_{p(\cdot)}^{\alpha(\cdot), q} F_{\beta}^{s}\left(\mathbb{R}^{n}, \Psi\right)}^{(4)} \cdot
$$

Step 3. Choosing $t=1$ in Step 1 and omitting the integration over $t$ we see immediately

$$
\|f\|_{K_{p(\cdot)}^{\alpha(\cdot), q} F_{\beta}^{s}\left(\mathbb{R}^{n}\right)}^{(5)} \lesssim\|f\|_{K_{p(\cdot)}^{\alpha(\cdot),} F_{\beta}^{s}\left(\mathbb{R}^{n)}\right.}^{(4)} \lesssim\|f\|_{K_{p(\cdot)}^{\alpha(\cdot), q} F_{\beta}^{s}\left(\mathbb{R}^{n}\right)}^{(5)}
$$

Step 4. What remains is to show that (3.19) is equivalent to the rest. 
First, let us prove that for any $f \in \mathcal{S}\left(\mathbb{R}^{n}\right)$

$$
\|f\|_{K_{p(\cdot)}^{\alpha(\cdot), q} F_{\beta}^{s}\left(\mathbb{R}^{n}\right)}^{(2)} \lesssim\|f\|_{K_{p(\cdot)}^{\alpha(\cdot), q} F_{\beta}^{s}\left(\mathbb{R}^{n}\right)}^{(3)} .
$$

From [35], for $0<r<\min \left\{p_{-}, \beta\right\}$, there exists a positive constant $C$ for any $f \in \mathcal{S}\left(\mathbb{R}^{n}\right)$

$$
\begin{aligned}
& \left(\int_{1}^{2}\left|\left(\Psi_{2^{-l} t}^{*} f\right)_{a}(x)\right|^{\beta} \frac{d t}{t}\right)^{r / \beta} \\
& \quad \leqslant C \sum_{k \in \mathbb{N}_{0}} 2^{-k N s} 2^{(k+l) n} \int_{\mathbb{R}^{n}} \frac{\left(\int_{1}^{2} \int_{|z|<2^{-(k+l)} t}\left|\left(\left(\Phi_{k+l}\right)_{t} * f\right)(z+y)\right|^{\beta} d z\left(d t / t^{n+1}\right)\right)^{r / \beta}}{\left(1+2^{l}|x-y|\right)^{a r}} d y .
\end{aligned}
$$

If $a r>n$ then we have

$$
g_{l}(y)=\frac{2^{n l}}{\left(1+2^{l}|y|\right)^{a r}} \in L_{1}\left(\mathbb{R}^{n}\right)
$$

Thus we have

$$
\begin{aligned}
& \left(\int_{1}^{2}\left|2^{l s}\left(\Phi_{2^{-l} t}^{*} f\right)_{a}(x)\right|^{\beta} \frac{d t}{t}\right)^{r / \beta} \\
& \quad \lesssim \sum_{k \in \mathbb{N}_{0}} 2^{-k N r} 2^{k n} 2^{l s r}\left[g_{l} *\left(\int_{1}^{2} \int_{|z|<2^{-(k+l) t}}\left|\left(\left(\Phi_{k+l}\right)_{t} * f\right)(z+\cdot)\right|^{\beta} d z \frac{d t}{t^{n+1}}\right)^{r / \beta}\right](x) .
\end{aligned}
$$

Now we use the majorant property of the Hardy-Littlewood maximal operator in [38] and continue estimating

$$
\begin{aligned}
& \left(\int_{1}^{2}\left|2^{l s}\left(\Phi_{2^{-l} t}^{*} f\right)_{a}(x)\right|^{\beta} \frac{d t}{t}\right)^{r / \beta} \\
& \quad \lesssim \sum_{k \in \mathbb{N}_{0}} 2^{l s r} 2^{k(-N r+n)} \mathcal{M}\left[\left(\int_{1}^{2} \int_{|z|<2^{-(k+l)} t}\left|\left(\left(\Phi_{k+l}\right)_{t} * f\right)(z+\cdot)\right|^{\beta} d z \frac{d t}{t^{n+1}}\right)^{r / \beta}\right](x) .
\end{aligned}
$$


An index shift on the right-hand side gives

$$
\begin{aligned}
& \left(\int_{1}^{2}\left|2^{l s}\left(\Phi_{2^{-l} t}^{*} f\right)_{a}(x)\right|^{\beta} \frac{d t}{t}\right)^{r / \beta} \\
& \quad \lesssim \sum_{k \in l+\mathbb{N}_{0}} 2^{l s r} 2^{(k-l)(-N r+n)} \mathcal{M}\left[\left(\int_{1}^{2} \int_{|z|<2^{-k} t}\left|\left(\left(\Phi_{k}\right)_{t} * f\right)(z+\cdot)\right|^{\beta} d z \frac{d t}{t^{n+1}}\right)^{r / \beta}\right](x) \\
& \quad=C \sum_{k \in l+\mathbb{N}_{0}} 2^{(l-k)(N r-n+r s)} 2^{k r s} \mathcal{M}\left[\left(\int_{1}^{2} \int_{|z|<2^{-k} t}\left|\left(\left(\Phi_{k}\right)_{t} * f\right)(z+\cdot)\right|^{\beta} d z \frac{d t}{t^{n+1}}\right)^{r / \beta}\right](x) .
\end{aligned}
$$

Then with analogous arguments as after (3.39), we obtain (3.47).

Second, we prove $\|f\|_{K_{p(r)}^{\alpha(), q} F_{\beta}^{s}\left(\mathbb{R}^{n)}\right.}^{(3)} \lesssim\|f\|_{K_{p(\cdot)}^{\alpha(\cdot)} F_{\beta}^{s}\left(\mathbb{R}^{n}\right)}^{(2)}$. Since for all $t>0$

$$
\begin{aligned}
\frac{1}{t^{n}} \int_{|z|<t}\left|\left(\Phi_{t} * f\right)(x+z)\right| d z & \lesssim \sup _{|z|<t} \frac{\left|\left(\Phi_{t} * f\right)(x+z)\right|}{(1+1 / t|z|)^{a}} \\
& \lesssim\left(\Phi_{t}^{*} f\right)_{a}(x) .
\end{aligned}
$$

Therefore we can obtain what we want. The proof is complete.

Remark 3.7. One can define homogenous spaces $K_{p(\cdot)}^{\alpha(\cdot), q} \dot{F}_{\beta}^{s}\left(\mathbb{R}^{n}\right), K_{p(\cdot)}^{\alpha(\cdot), q} \dot{B}_{\beta}^{s}\left(\mathbb{R}^{n}\right), \dot{K}_{p(\cdot)}^{\alpha(\cdot), q} \dot{F}_{\beta}^{s}\left(\mathbb{R}^{n}\right)$

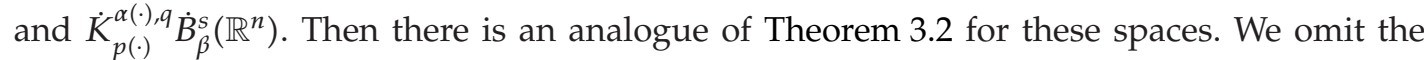
detail here and leave it for interested readers.

\section{Acknowledgments}

The authors would like to thank the referee for his carefully reading and suggestions which made the presentation more readable. J. Xu is supported by the National Natural Science Foundation of China (Grant no. 11071064 and 11226167) and the Natural Science Foundation of Hainan Province (no. 111006) and the Scientific Research Foundation of Hainan Province Education Bureau (no. Hjkj2011-19).

\section{References}

[1] O. Kováčik and J. Rákosník, “On spaces $L^{p(x)}$ and $w^{k, p(x)}$, , Czechoslovak Mathematical Journal, vol. 41, no. 4, pp. 592-618, 1991.

[2] A. Almeida and P. Hästö, "Besov spaces with variable smoothness and integrability," Journal of Functional Analysis, vol. 258, no. 5, pp. 1628-1655, 2010.

[3] A. Almeida, J. Hasanov, and S. Samko, "Maximal and potential operators in variable exponent Morrey spaces," Georgian Mathematical Journal, vol. 15, no. 2, pp. 195-208, 2008.

[4] L. Diening, P. Hästö, and S. Roudenko, "Function spaces of variable smoothness and integrability," Journal of Functional Analysis, vol. 256, no. 6, pp. 1731-1768, 2009.

[5] P. Gurka, P. Harjulehto, and A. Nekvinda, "Bessel potential spaces with variable exponent," Mathematical Inequalities E Applications, vol. 10, no. 3, pp. 661-676, 2007. 
[6] H. Kempka, "2-microlocal Besov and Triebel-Lizorkin spaces of variable integrability," Revista Matemática Complutense, vol. 22, no. 1, pp. 227-251, 2009.

[7] H. Kempka, "Atomic, molecular and wavelet decomposition of generalized 2-microlocal Besov spaces," Journal of Function Spaces and Applications, vol. 8, no. 2, pp. 129-165, 2010.

[8] P. A. Hästö, "Local-to-global results in variable exponent spaces," Mathematical Research Letters, vol. 16, no. 2, pp. 263-278, 2009.

[9] J. Xu, "Variable Besov and Triebel-Lizorkin spaces," Annales Academix Scientiarum Fennicx. Mathematica, vol. 33, no. 2, pp. 511-522, 2008.

[10] J. S. Xu, "The relation between variable Bessel potential spaces and Triebel-Lizorkin spaces," Integral Transforms and Special Functions, vol. 19, no. 7-8, pp. 599-605, 2008.

[11] D. Cruz-Uribe, A. Fiorenza, J. M. Martell, and C. Pérez, "The boundedness of classical operators on variable $L^{p}$ spaces," Annales Academiæ Scientiarum Fennicæ. Mathematica, vol. 31, no. 1, pp. 239-264, 2006.

[12] D. Cruz-Uribe, A. Fiorenza, and C. J. Neugebauer, "The maximal function on variable $L^{p}$ spaces," Annales Academix Scientiarum Fennicæ. Mathematica, vol. 28, no. 1, pp. 223-238, 2003.

[13] L. Diening, P. Harjulehto, P. Hästö, and M. Růžička, Lebesgue and Sobolev Spaces with Variable Exponents, vol. 2017 of Lecture Notes in Mathematics, Springer, Heidelberg, Germany, 2011.

[14] P. Harjulehto, P. Hästö, U. V. Le, and M. Nuortio, "Overview of differential equations with nonstandard growth," Nonlinear Analysis: Theory, Methods E Applications, vol. 72, no. 12, pp. 4551-4574, 2010.

[15] S. Lu, D. Yang, and G. Hu, Herz Type Spaces and Their Applications, Science Press, Beijing, China, 2008.

[16] E. Hernández and D. Yang, "Interpolation of Herz spaces and applications," Mathematische Nachrichten, vol. 205, pp. 69-87, 1999.

[17] S. Lu, K. Yabuta, and D. Yang, "Boundedness of some sublinear operators in weighted Herz-type spaces," Kodai Mathematical Journal, vol. 23, no. 3, pp. 391-410, 2000.

[18] X. Li and D. Yang, "Boundedness of some sublinear operators on Herz spaces," Illinois Journal of Mathematics, vol. 40, no. 3, pp. 484-501, 1996.

[19] S. Lu, "Multipliers and Herz type spaces," Science in China. Series A, vol. 51, no. 10, pp. 1919-1936, 2008.

[20] S. Z. Lu and D. C. Yang, "The decomposition of weighted Herz space on $\mathbb{R}^{n}$ and its applications," Science in China, vol. 38, no. 2, pp. 147-158, 1995.

[21] J. Xu, "A discrete characterization of Herz-type Triebel-Lizorkin spaces and its applications," Acta Mathematica Scientia. Series B, vol. 24, no. 3, pp. 412-420, 2004.

[22] J. Xu, "Equivalent norms of Herz-type Besov and Triebel-Lizorkin spaces," Journal of Function Spaces and Applications, vol. 3, no. 1, pp. 17-31, 2005.

[23] J. Xu and D. Yang, "Applications of Herz-type Triebel-Lizorkin spaces," Acta Mathematica Scientia. Series B, vol. 23, no. 3, pp. 328-338, 2003.

[24] J. S. Xu and D. C. Yang, "Herz-type Triebel-Lizorkin spaces. I," Acta Mathematica Sinica, vol. 21, no. 3, pp. 643-654, 2005.

[25] M. Izuki, "Boundedness of sublinear operators on Herz spaces with variable exponent and application to wavelet characterization," Analysis Mathematica, vol. 36, no. 1, pp. 33-50, 2010.

[26] M. Izuki, "Boundedness of commutators on Herz spaces with variable exponent," Rendiconti del Circolo Matematico di Palermo. Second Series, vol. 59, no. 2, pp. 199-213, 2010.

[27] M. Izuki, "Vector-valued inequalities on Herz spaces and characterizations of Herz-Sobolev spaces with variable exponent," Glasnik Matematicki, vol. 45, no. 2, pp. 475-503, 2010.

[28] C. Shi and J. Xu, "Herz type Besov and Triebel-Lizorkin spaces with variable exponents," Frontiers of Mathematics in China. to appear.

[29] C. Shi and J. Xu, "A characterization of Herz-Besov-Triebel spaces with variable exponent," Acta Mathematica Sinica, vol. 55, no. 4, pp. 653-664, 2012.

[30] A. Almeida and D. Drihem, "Maximal, potential and singular type operators on Herz spaces with variable exponents," Journal of Mathematical Analysis and Applications, vol. 394, no. 2, pp. 781-795, 2012.

[31] L. Diening, "Maximal function on generalized Lebesgue spaces $L^{p(x)}$," Mathematical Inequalities $\mathcal{E}$ Applications, vol. 7, no. 2, pp. 245-253, 2004.

[32] A. Nekvinda, "Hardy-Littlewood maximal operator on $L^{p(x)}$, " Mathematical Inequalities \& Applications, vol. 7, no. 2, pp. 255-265, 2004.

[33] L. Tang and D. Yang, "Boundedness of vector-valued operators on weighted Herz spaces," Approximation Theory and Its Applications, vol. 16, no. 2, pp. 58-70, 2000. 
[34] J. Peetre, “On spaces of Triebel-Lizorkin type," Arkiv för Matematik, vol. 13, pp. 123-130, 1975.

[35] T. Ullrich, "Continuous characterizations of Besov-Lizorkin-Triebel spaces and new interpretations as coorbits," Journal of Function Spaces and Applications, vol. 2012, Article ID 163213, 47 pages, 2012.

[36] V. S. Rychkov, "On a theorem of Bui, Paluszyński, and Taibleson," Proceedings of the Steklov Institute of Mathematics, vol. 227, no. 18, pp. 286-298, 1999.

[37] M. Frazier and B. Jawerth, "Decomposition of Besov spaces," Indiana University Mathematics Journal, vol. 34, no. 4, pp. 777-799, 1985.

[38] E. M. Stein and G. Weiss, Introduction to Fourier analysis on Euclidean spaces, Princeton University Press, Princeton, NJ, USA, 1971. 


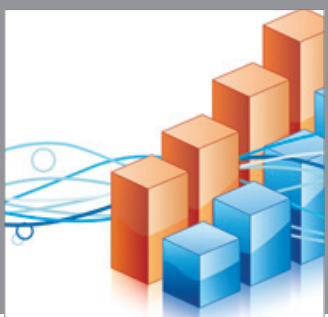

Advances in

Operations Research

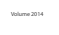

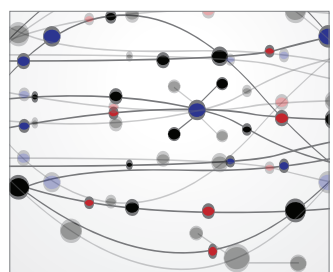

\section{The Scientific} World Journal
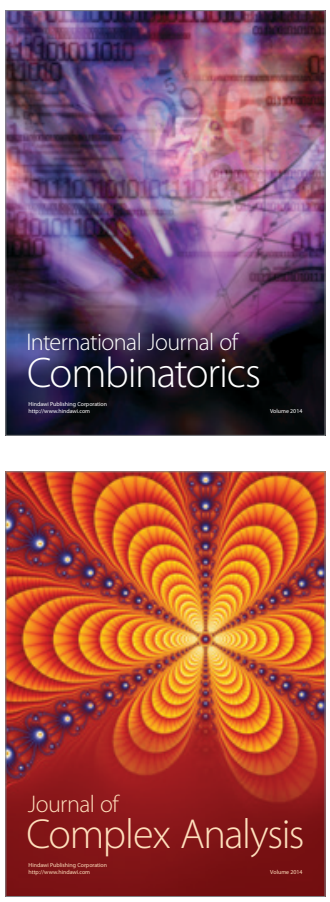

International Journal of

Mathematics and

Mathematical

Sciences
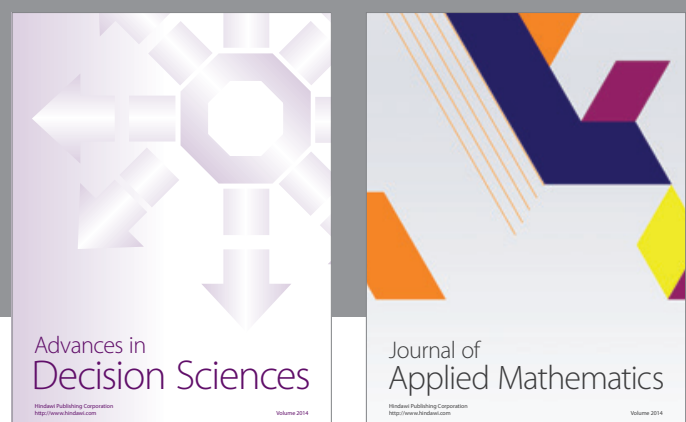

Journal of

Applied Mathematics
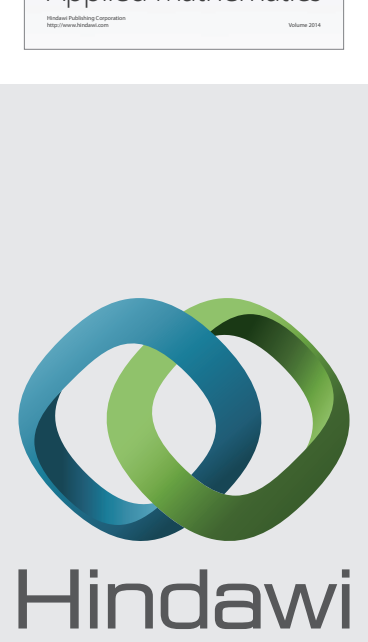

Submit your manuscripts at http://www.hindawi.com
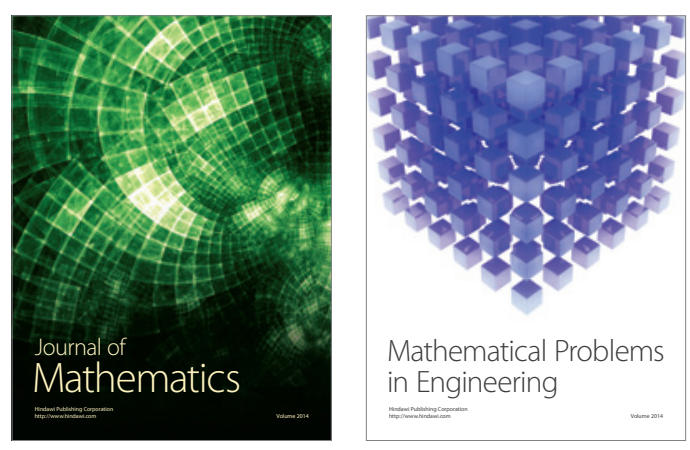

Mathematical Problems in Engineering
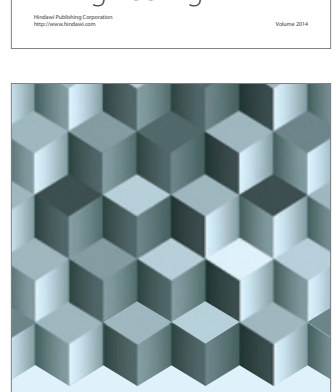

Journal of

Function Spaces
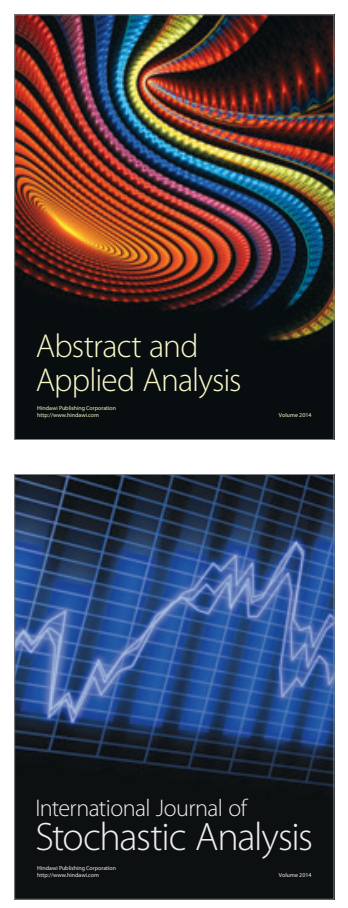

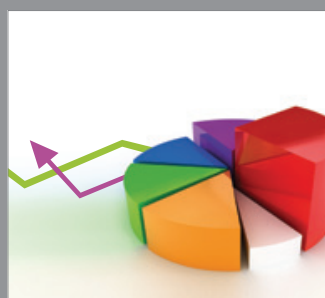

ournal of

Probability and Statistics

Promensencen
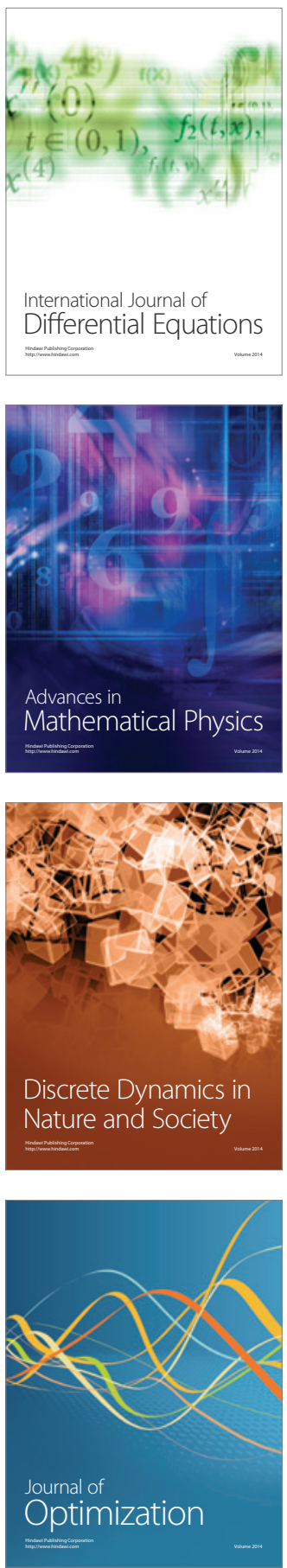